ARTICLE

Received 10 Aug 2015 | Accepted 24 May 2016 | Published 27 Jun $2016 \quad$ DOI: 10.1038/ncomms12042 OPEN

\title{
Stimulus-responsive light-harvesting complexes based on the pillararene-induced co-assembly of $\beta$-carotene and chlorophyll
}

Yan Sun ${ }^{1}$, Fang Guo ${ }^{1}$, Tongfei Zuo ${ }^{1}$, Jingjing Hua ${ }^{1} \&$ Guowang Diao ${ }^{1}$

The locations and arrangements of carotenoids at the subcellular level are responsible for their designated functions, which reinforces the necessity of developing methods for constructing carotenoid-based suprastructures beyond the molecular level. Because carotenoids lack the binding sites necessary for controlled interactions, functional structures based on carotenoids are not easily obtained. Here, we show that carotene-based suprastructures were formed via the induction of pillararene through a phasetransfer-mediated host-guest interaction. More importantly, similar to the main component in natural photosynthesis, complexes could be synthesized after chlorophyll was introduced into the carotene-based suprastructure assembly process. Remarkably, compared with molecular carotene or chlorophyll, this synthesized suprastructure exhibits some photocatalytic activity when exposed to light, which can be exploited for photocatalytic reaction studies of energy capture and solar conversion in living organisms.

\footnotetext{
${ }^{1}$ College of Chemistry and Chemical Engineering, Yangzhou University, Yangzhou, Jiangsu 225002, China. Correspondence and requests for materials should be addressed to G.D. (email: gwdiao@yzu.edu.cn).
} 
C arotenoids are a diverse group of structurally related compounds ${ }^{1}$ that perform various functions in living organisms $^{2}$. Their conjugated double-bond systems determine their photochemical properties and their chemical reactivities $^{3}$, which, in turn, influence the properties of these subcellular structures ${ }^{4}$. They are relevant to human and animal processes related to nutrition ${ }^{5}$, immune systems ${ }^{6}$ and antioxidation ${ }^{7}$. Their roles in plants and microorganisms are ascribed mostly to acting as a stabilizing structure for light-harvesting complexes (LHCs) ${ }^{8}$, photoprotection $^{9}$, absorbing light and transferring energy to chlorophyll pigments ${ }^{10}$, which acts as a driving force for photosynthesis ${ }^{1}$. Furthermore, the molecular geometry and specific interactions with other molecules in the structures are critical for ensuring that the carotenoids fit into the suprastructures in the correct location and orientation ${ }^{12}$. Considerable effort has been devoted to investigating the biochemistry and molecular biology of carotenoids $^{13}$. Nevertheless, in the fields of both nutrition utilization and suprastructure construction, suprastructure development has been limited by the hydrophobic nature of carotenoids ${ }^{14}$.

Recently, in the carotenoid-related fields, this problem has been partially solved through the use of hydrophilic vectors ${ }^{15}$. For example, natural carotene has been wrapped into hydrophilic vectors by spinning disk processing to construct a caroteneloaded particle ${ }^{16}$. Notably, supramolecular studies have been focused primarily on the characterization of the natural photosynthesis machinary ${ }^{17}$. The design and synthesis of programmable entities based on carotenoids still faces many issues due to the low solubility of natural pigments in aqueous media. Thus, no carotenoid molecules have been introduced as building blocks to form suprastructures that mimic natural structures. To solve these problems, synthetic water-soluble analogues have often been used instead of natural pigments as building units ${ }^{18}$. Compared with the successful fabrication of many synthetic supramolecular systems based on hydrophilic biomolecules, including peptides ${ }^{19}$, fatty acids $^{20}$, nucleotides ${ }^{21}$ and porphyrins ${ }^{22}$, the preparation of synthetic suprastructures based on hydrophobic biomolecules remains in the initial stages. Therefore, the development of effective strategies for assembling carotenoids into intricate and customizable large-scale systems that perform the same physiological functions as they perform in vivo is now essential ${ }^{23}$.

Fortunately, supramolecular chemistry provides a new framework to tackle this difficult question. Thus far, supramolecular macrocycles, such as crown ethers ${ }^{24-26}$, cyclodextrins ${ }^{27}$ and calixarenes 28,29 , have been used for various applications. Pillararenes ${ }^{30,31}$, which are composed of hydroquinone units linked by methylene bridges at the para positions, are an especially interesting new class of macrocyclic host in supramolecular chemistry. Their unique, intrinsically rigid and symmetrical pillar architecture endows them with an outstanding ability to selectively bind various types of guest molecules ${ }^{32}$. This property has been used to construct various interesting supramolecular systems ${ }^{33}$, including nanomaterials ${ }^{34}$, sensors ${ }^{35}$, ion channels ${ }^{36}$ and drug-delivery systems ${ }^{37}$.

In this article, $\beta$-carotene $(\beta$-CAR) was selected as a type of carotenoid to represent the carotenoid family. A synthetic water-soluble carboxyl-modified pillararene (WP5) was also chosen because its cavity dimensions are appropriate for the accommodation of $\beta$-CAR, which could further induce the construction of a $\beta$-CAR-based substructure. We envisioned that $\beta$-CAR might bind WP5 in water to achieve WP5 $\supset \beta$-CAR complexation (WCC) via the hydrophobic effect. Then, the resulting amphiphilic WCC might be able to form a $\beta$-CAR-containing suprastructure in water via self-assembly.
On the basis of the fascinating properties exhibited by natural chlorophyll/carotenoid complexes in photosynthesis, chlorophyll-b (Chl-b) was selected as the co-assembly factor participating in the preparation process with WCC, and a similar suprastructure of Chl-b-containing LHC was synthesized. These hydrophilic complexes possess a suite of unusual properties, including spontaneous growth, fusion, $\mathrm{pH}$ stimulus responsiveness and even some photocatalytic activity.

\section{Results}

Host-guest interaction between WP5 and $\beta$-CAR. The pillar[5] arene carboxyl derivative WP5 was prepared according to published procedures ${ }^{38}$ and was identified by ${ }^{1} \mathrm{H}-\mathrm{NMR}$ spectroscopy after being dissolved in $\mathrm{D}_{2} \mathrm{O}$. According to the results of Gaussian 09 calculations, the internal diameter of the cavity is $0.5 \mathrm{~nm}$ (ref. ${ }^{32}$ and the length of the WP5 is $1.1 \mathrm{~nm}$. Within the as-prepared synthetic LHC systems, the role of WP5 (Fig. 1b) is primarily to improve the solubility of $\beta$-CAR in aqueous environments and to further induce the hierarchical arrangement of $\beta$-CAR during assembly. $\beta$-CARs are naturally abundant pigments that are extremely hydrophobic and have low solubility in water. As shown in the energy-minimized structure of $\beta$-CAR (Fig. 1c), the length and width of $\beta$-CAR are $\sim 3.0$ and $0.5 \mathrm{~nm}$, respectively. A remarkable feature of $\beta$-CAR is that its molecular shape and size (a slight twist of conformation) fit well within the cavity of WP5, which provides the molecular basis for the interaction between WP5 and $\beta$-CAR. $\beta$-CAR units serve as the structural skeleton, providing photoprotective functions and binding sites for Chl-b (Fig. 1d). Chl-b molecules serve as the light-absorbing and energy-transferring components; they can attach to the hydrophobic domains by inserting their long alkyl chains into WCC. Given that the porphyrin head group of Chl-b exhibits photocatalytic activity, it is a rational choice for a functional component for integrating the photoprotective properties of $\beta$-CAR. The length of the hydrophobic tail of Chl-b $(2.4 \mathrm{~nm})$ matches the length of the hydrophobic moieties of WCC $(2.3 \mathrm{~nm})$ well. Thus, the hydrophobic tail of Chl-b could be used to anchor Chl-b to the hydrophobic segments of WCC and further facilitate the investigation of photosynthesis on the supramolecular level. In addition, WP5 as the carboxylic sodium salt and its precipitation from water as the corresponding carboxylic acid could be reversibly adjusted by changing the $\mathrm{pH}$ of the solution, which could endow the proposed suprastructure with $\mathrm{pH}$ responsiveness. The samples were prepared by a simple procedure. $\beta$-CAR powder was first dissolved in ethanol, and then, $2 \mathrm{ml}$ of this $\beta$-CAR solution $(150 \mu \mathrm{M})$ was added to $2 \mathrm{ml}$ of a dilute sodium hydroxide aqueous solution of WP5 $(150 \mu \mathrm{M})$ using a pipette, without stirring. The formation of WCC (Fig. 2a) is likely to be mainly driven by hydrophobic interactions between the hydrophobic cavity of WP5 and the lipophilic $\beta$-CAR. As shown in Fig. 2b, a 'tadpole-like' WCC with the bulky group at one side of $\beta$-CAR was threaded into the cavity of WP5, and the residue of $\beta$-CAR protruding from the WP5 cavity. The TCH (ten-carboxylic acid head) segment is the hydrophilic region with an extended length of $1.1 \mathrm{~nm}$, and the SCT (single-carotene tail) is the segment $(2.3 \mathrm{~nm})$ that possesses a long hydrophobic tail that imparts amphiphilic character to WCC. The complexation between WP5 and $\beta$-CAR was first demonstrated by ${ }^{1} \mathrm{H}-\mathrm{NMR}$ spectroscopy. As shown in Supplementary Figs 1, 2, the proton peaks $\mathrm{H}_{3}$ of the methylene moieties at both rims were split into quartets from singlets, likely because of the inclusion of terpene in the cavity of WP5 restricted the swinging of the constituent units, which led to the loss of internal symmetry of $\mathrm{H}_{3}$ protons. At the same time, downfield shifts of the aromatic protons $\mathrm{H}_{1}$ and $\mathrm{H}_{2}$ of 
a

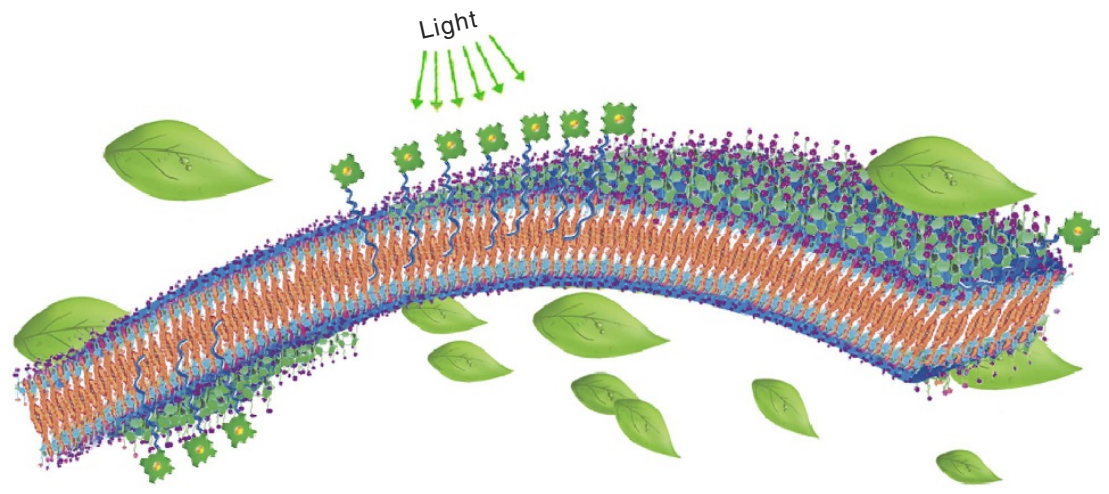

b

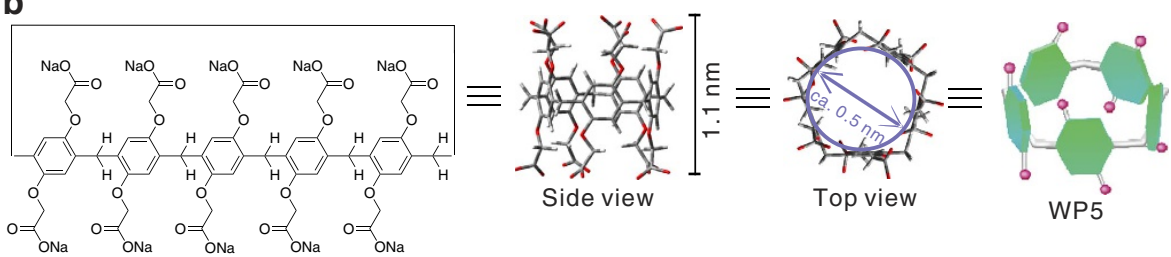

C

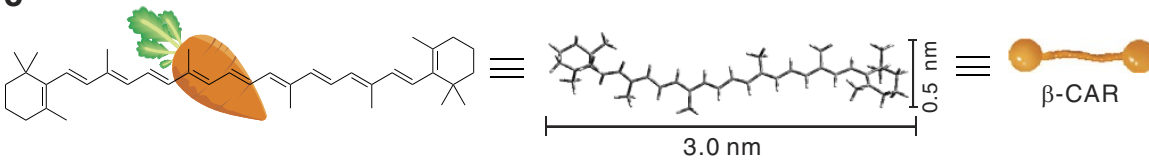

d

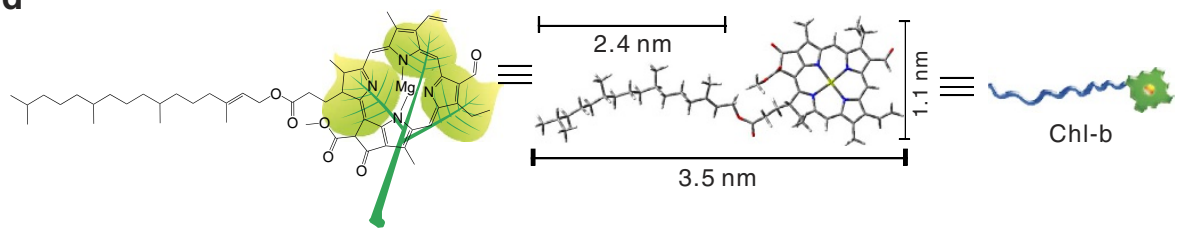

Figure 1 | Structural model of building blocks. (a) LHC containing Chl-b formed by (b) WP5, (c) $\beta$-CAR and (d) Chl-b. For clarity, the $\beta$-CAR-based hydrophobic interior layer is orange, and the WP5-based hydrophilic exterior layer is green.

WP5 were observed. In contrast, remarkable upfield chemical shifts of the methylene protons $\left(\mathrm{H}_{\mathrm{b}}, \mathrm{H}_{\mathrm{c}}\right.$ and $\left.\mathrm{H}_{\mathrm{d}}\right)$ and methyl protons $\left(\mathrm{H}_{\mathrm{a}}\right.$ and $\left.\mathrm{H}_{\mathrm{e}}\right)$ of the bulky terpene were observed $\quad\left(\Delta \delta\left(\mathrm{H}_{\mathrm{b}}\right)=0.228\right.$ p.p.m., $\quad \Delta \delta\left(\mathrm{H}_{\mathrm{c}}\right)=0.259$ p.p.m., $\Delta \delta\left(\mathrm{H}_{\mathrm{d}}\right)=0.354$ p.p.m., $\quad \Delta \delta\left(\mathrm{H}_{\mathrm{a}}\right)=0.160$ p.p.m. and $\Delta \delta\left(\mathrm{H}_{\mathrm{e}}\right)=$ 0.150p.p.m.) on the addition of WP5 due to the shielding effect of the electron-rich cavities of WP5 toward $\beta$-CAR, which clearly demonstrated the inclusion of the terpene section of $\beta$-CAR into the hydrophobic WP5 cavity. Moreover, the signals derived from protons $\mathrm{H}_{\mathrm{g}}\left(\Delta \delta\left(\mathrm{H}_{\mathrm{g}}\right)=-0.027\right.$ p.p.m. $)$ and methyl protons $\left(\mathrm{H}_{\mathrm{f}}\right)$ on linear unsaturated carbon chain shifted from 6.1-6.8 p.p.m. to downfield 6.6-6.3 p.p.m. $\left(\Delta \delta\left(\mathrm{H}_{\mathrm{f}}\right)=-0.5\right.$ p.p.m. $)$. A similar phenomenon was also observed for the inclusion complexation between water-soluble pillar[5]arene and guest in previous reports 39,40 . The above results revealed that the cavity was fully threaded by $\beta$-CAR with the protons $\mathrm{H}_{\mathrm{a}}, \mathrm{H}_{\mathrm{b}}, \mathrm{H}_{\mathrm{c}}, \mathrm{H}_{\mathrm{d}}$ and $\mathrm{H}_{\mathrm{e}}$ in the hydrophobic WP5 cavity and that the other protons on a linear unsaturated carbon chain $\left(\mathrm{H}_{\mathrm{f}}\right.$, and $\left.\mathrm{H}_{\mathrm{g}}\right)$ protruded out of the cavity. The binding affinity for such host-guest inclusion might be mainly driven by the hydrophobic interactions. The generation of $\beta$-CAR-based WCC was then supported by Raman spectroscopy (Fig. 2c). The features of WCC in the $1,600-900 \mathrm{~cm}^{-1}$ region are similar to those of the pure $\beta$-CAR spectra $^{41}$ : the peak at $1,526 \mathrm{~cm}^{-1}$ originates from the stretching modes of the conjugated $\mathrm{C}=\mathrm{C}$ bonds; the peak at $1,160 \mathrm{~cm}^{-1}$ arises from a mixture of $\mathrm{C}=\mathrm{C}$ and $\mathrm{C}-\mathrm{C}$ bond stretching modes with $\mathrm{C}-\mathrm{H}$ bending modes; and the peak at $1,009 \mathrm{~cm}^{-1}$ is attributed to the stretching modes of $\mathrm{C}-\mathrm{CH}_{3}$ bonds between the main chain and the side methyl carbons. These peaks indicate that $\beta$-CAR is present in the WCC. In addition, the $v_{1}$ band assigned to $\mathrm{C}=\mathrm{C}$ stretching (which is sensitive to host-guest interactions) blue-shifted $\left(5 \mathrm{~cm}^{-1}\right)$ from 1,521 to $1,526 \mathrm{~cm}^{-1}$, which not only provided further direct evidence of host-guest interactions between WP5 and $\beta$-CAR, but also further confirmed that the bulky groups at the ends of the $\beta$-CAR units were inserted into the cavity ${ }^{42}$.

The encapsulation of a guest $(\mathrm{G})$ in the hydrophobic cavity might also influence the polarizability of the host $(\mathrm{H})$ cavity $^{43}$, which can be characterized by the fluorescence spectra. As shown in Fig. 2d, different $(\mathrm{G}) /(\mathrm{H})$ mole ratios $((\mathrm{H})=150 \mu \mathrm{M})$ demonstrated that the confinement of $\beta$-CAR in the cavity strongly affects the fluorescence behaviour. The stoichiometry of the WCC between WP5 and $\beta$-CAR was further investigated based on the fluorescence spectra. The peak at $653 \mathrm{~nm}$ was selected for the investigation, which revealed a 1:1 binding stoichiometry between WP5 and $\beta$-CAR (Fig. 2e). Notably, the peak at $654 \mathrm{~nm}$ was selected because of the emission peak overlap of WP5 and $\beta$-CAR at $334 \mathrm{~nm}$ (Supplementary Fig. 3a-f). Furthermore, the fluorescence spectra of $(\mathrm{G}) /(\mathrm{H})$ at a higher host concentration $((\mathrm{H})=200 \mu \mathrm{M})$ were also monitored, as also shown in (Supplementary Fig. $3 \mathrm{~g}-\mathrm{i}$ ). The peak at $375 \mathrm{~nm}$ was selected for the investigation of the intensity change of $\beta$-CAR 
a

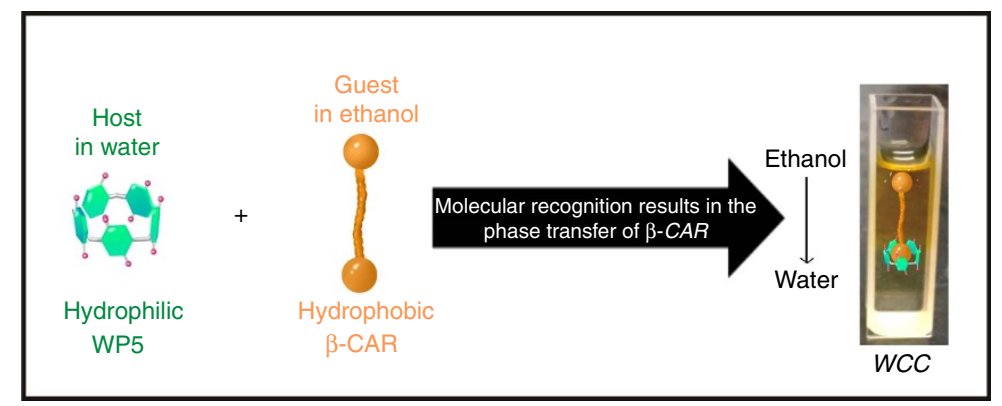

c

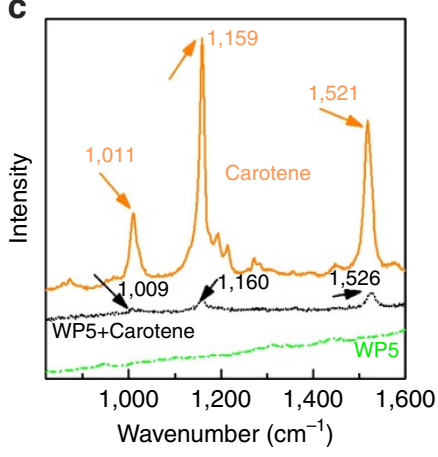

f

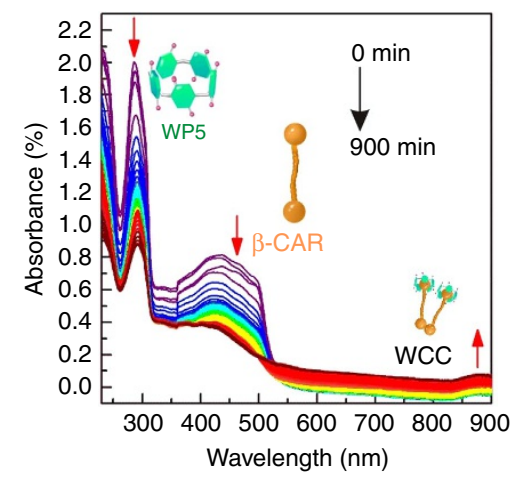

b

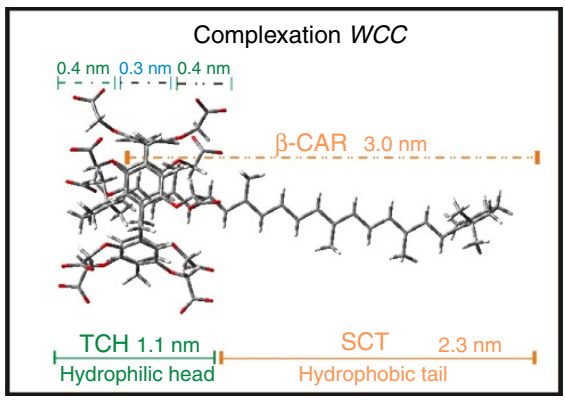

e

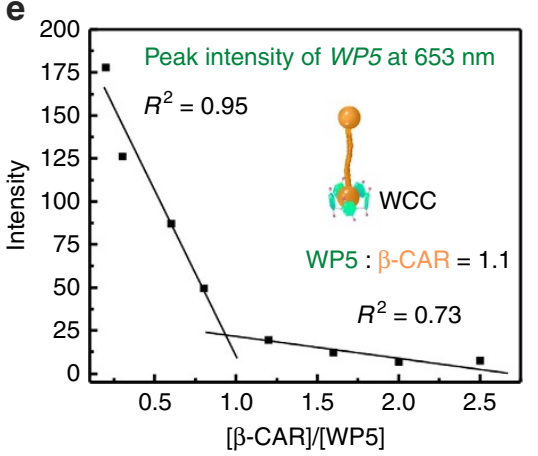

h

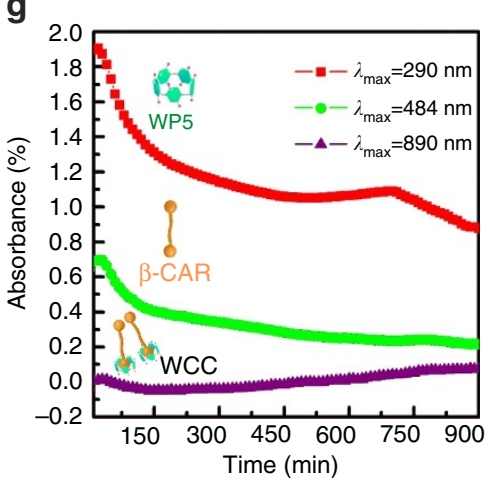

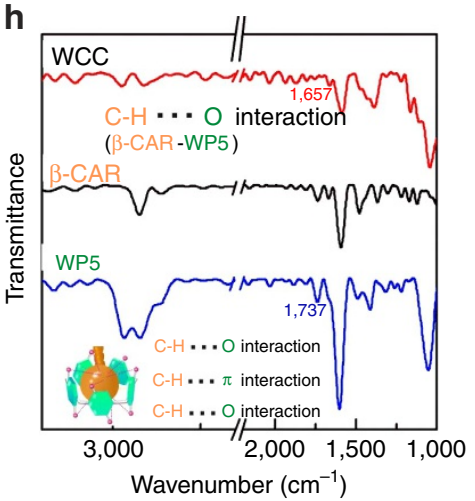

Figure 2 | Synthesis of the WCC. (a) A schematic displaying the synthetic route to the WCC. (b) Simulated structure of the WCC. (c) Raman spectra of $\beta$-CAR and the WCC. (d) Fluorescence spectra of WP5 $(150 \mu \mathrm{M})$ on addition of $\beta$-CAR in ethanol/water (excited at $268 \mathrm{~nm}$ ) at room temperature.

(e) The fluorescence intensity changes of WP5 at $653 \mathrm{~nm}$. (f) Time-dependent ultraviolet-visible spectra showing the formation of the WCC, as indicated by the changes in the intensities of the peaks at 290 and $484 \mathrm{~nm}$ (20 min each). (g) Plot of absorbance intensity as a function of time (20 min each).

(h) FT-IR spectra of WP5, $\beta$-CAR and the WCC.

(this peak could not be observed when the WP5 concentration was $150 \mu \mathrm{M}$, which resulted in a relatively low concentration of $\beta$-CAR), and the peak at $653 \mathrm{~nm}$ was selected for the investigation of the intensity change of WP5. The results of both of these experiments further demonstrated that the $(\mathrm{H}) /(\mathrm{G})$-binding stoichiometry is 1:1 (ref. 34). According to the fluorescence intensity change in Fig. $2 \mathrm{~d}$, the association constant $\left(K_{\mathrm{a}}\right)$ of WP5 $\supset \beta$-CAR was calculated to be $(2.34 \pm 2.06) \times 10^{5} \mathrm{M}^{-1}$ using a non-linear curve-fitting method (Supplementary Fig. 3j). Aside from the above fluorescence results, the stoichiometry of complexation for WP5 $\supset \beta$-CAR was further proven by Job's plot method using ultraviolet-visible spectroscopy, which confirmed the 1:1 binding stoichiometry for WP5 $\supset \beta$-CAR complexation (Supplementary Fig. 4$)^{44}$. To gain further insight into the dynamic interaction between WP5 and $\beta$-CAR, we acquired 45 sequential ultraviolet-visible spectra at 20-min intervals for the same sample. As shown in Fig. $2 \mathrm{f}$, the intensities of the characteristic peaks at $\sim 290 \mathrm{~nm}$ and $484 \mathrm{~nm}$ (attributed to the absorbance of WP5 and $\beta$-CAR, respectively) decreased from 0 to $900 \mathrm{~min}$. Meanwhile, the emergence and continued increase in intensity of a peak at $890 \mathrm{~nm}$ were observed, providing another piece of evidence for the formation of the WCC. Figure $2 \mathrm{~g}$ clearly shows that the increase in intensity of the peak at $890 \mathrm{~nm}$ is associated with a concomitant decrease in the intensities of the features at $290 \mathrm{~nm}$ and $484 \mathrm{~nm}$, which might be caused by the formation of microaggregates (Supplementary Fig. 5) and leads to the decrease of WP5 and $\beta$-CAR absorption intensity. Further evidence for the existence of the WCC was obtained by Fourier-transform infrared (FT-IR) spectroscopy, which shows that hydrogen bonds formed in the WCC (between the $-\mathrm{COO}^{-}$of WP5 and the $\mathrm{CH}_{3}-$ of $\beta$-CAR). As shown in Fig. 2h, in the spectrum of the WP5 before self-assembly, the absorbance at $1,737 \mathrm{~cm}^{-1}$ is attributed to a non-hydrogen-bonded - $\mathrm{COOH}$ group, which implies the presence of free carboxyl groups ${ }^{45}$. In contrast, this stretching vibration at $1,737 \mathrm{~cm}^{-1}$ disappeared after the complexation. 
These results show that strong and orderly hydrogen bonds were formed in the WCC.

Construction of HMS based on the WCCs. WCCs were then utilized as building blocks in the construction of light-harvesting antenna complexes (LHCs). After the as-prepared solutions were aged for 7 days, orange aggregates appeared in the solutions. As shown in Fig. 3a (Supplementary Fig. 6), the aggregates were determined to be hollow microspheres (HMSs) based on optical microscopy (OM) observations, and the orange colour of the wall confirmed the homogeneous distribution of $\beta$-CAR across the HMSs. The DLS result showed that the WP5 $\supset \beta$-CAR-based HMS had an average diameter of $1,855 \mathrm{~nm}$ (Supplementary Fig. 5b). Autofluorescence is exhibited in Fig. 3b-d (Supplementary Fig. 7) and is attributed to the presence of the conjugated system in $\beta$-CAR and WP5. Scanning electron microscopy (SEM) micrographs further confirmed the microspherical morphology of the HMSs (Fig. 3e, Supplementary Fig. 8). The elemental mapping analysis demonstrated the homogeneous distribution of $\mathrm{O}$ and $\mathrm{Na}$, which are characteristic elements in WP5 (Fig. 3f), across the HMSs, providing powerful evidence that the HMSs are composed of WP5. Interestingly, an HMS with a partially broken shell was observed (Fig. 3g) by SEM. The layer peeled off in the form of an arc, which indicated that the HMS might be composed of concentric multilayers (the observed wall thickness is ca. $40 \mathrm{~nm}$ ). Furthermore, the marked red-shift of the ultraviolet-visible spectra suggested that J-aggregates had been formed (Fig. $3 \mathrm{~h})^{46}$, indicating that the $\beta$-CARs are oriented in the WCC bilayer with the long axis almost perpendicular to the bilayer surface and with the two WP5 located in the hydrophilic regions on both sides of the bilayer. Strong anisotropic photoluminescence (birefringence) was observed when the dispersion was placed between crossed polarizers (Fig. 3i, Supplementary Fig. 9), providing further evidence for the presence of ordered arrays of WP5 and $\beta$-CAR within the HMS. Transmission electron microscopy (TEM) was used to characterize the fine structure of the HMSs. Figure $3 j$ shows a bright-field TEM micrograph of one HMS. The intensity of the central part of the HMS is much higher than that of the edge, indicating that the sphere is hollow. The thickness of the microspherical wall was calculated to be $\sim 100 \mathrm{~nm}$ based on this TEM image. The extended geometries of the $\beta$-CAR and their 1:1 complexation with the WCC are depicted Fig. 3k. Given that the maximal length of the WCC-based bilayer calculated using Gaussian is $\sim 4.6 \mathrm{~nm}$, this TEM observation suggests that the HMS may possess a multilayer structure. Notably, WP5s with ten carboxylic acids possess negative charges, which might result in the enhancement of electrostatic repulsion. However, similar to the multiwalled microtubule assemblies obtained in weakly acidic environments ${ }^{47,48}$, the carboxylic acid groups in this study are partially dissociated in weakly basic environments. Hydrogen bonds thus dominate the interactions within the HMS and further weaken the electrostatic repulsion, facilitating the formation of multilayer microspheres. The packing information with respect to the WCC was obtained from small-angle X-ray scattering (SAXS) data. As shown in Fig. 3l, a broad Bragg reflection peak centred at $0.20 \AA^{-1}$, corresponding to the ordered structure with an interlayer spacing of $\sim 3.1 \mathrm{~nm}$, was observed. Note that this broad peak covers the $q$ region range from 0.15 to $0.25 \AA^{-1}$, corresponding to an interlayer spacing between 2.5 to $4.2 \mathrm{~nm}$.

These distances were slightly shorter than the maximal calculated value $(4.6 \mathrm{~nm})$, possibly due to the conformational change or partial curling of the unsaturated carbon chain ${ }^{49}$. In addition, two slight peaks at lower $q$ values $\left(0.05\right.$ and $\left.0.1 \AA^{-1}\right)$ were observed in the profile, indicating that there are multiple orders of structure with an interlayer spacing of $\sim 3.1 \mathrm{~nm}$ in the HMS. However, these peaks are weak, possibly because of the partially inhomogeneous lamellar structure within the HMS. In addition, considering the SAXS results, we conjectured that an interlayer spacing centred at $3.1 \mathrm{~nm}$ may exist in the HMS. Furthermore, the zeta-potential of the HMS decreased from $\sim 0 \mathrm{mV}$ (pure $\beta$-CAR) to $-31.6 \mathrm{mV}$, which provides further evidence that the hydrophilic WP5 is located on the exterior surface of the HMS (Supplementary Fig. 10).

On the basis of the aforementioned results, we proposed the following mechanism for the formation of the WCC-based HMSs (Fig. 3m): The hydrophobic interactions between $\beta$-CAR and WP5 provide the driving force for the formation of host-guest complexation WCC, which results in the phase transfer of $\beta$-CAR from ethanol to water. Meanwhile, hydrogen bonding $(\mathrm{CH}-\mathrm{O})$ and the $\mathrm{CH}-\pi$ interaction ${ }^{50}$ between $\beta$-CAR and WP5 render the WCC stable in water. Subsequently, hydrophobic interactions associated with aromatic stacking ${ }^{51,52}$ drive the WCC to assemble into an HMS, in which the hydrophobic $\beta$-CARs are shielded from the water, while the hydrophilic WP5s are in contact with the aqueous environment.

Synthesis of HMS-based LHC containing Chl-b. Additional experiments were carried out to investigate the possibility of reproducing similar 'tadpole-like' host-guest complexes by using Chl-b instead of $\beta$-CAR. When Chl-b solution was added to WP5, the proton peaks $\mathrm{H}_{3}$ of the methylene moieties on both rims were split into quartets from singlets, likely because of the inclusion of Chl-b in the cavity of WP5, which affected the swinging of the constituent units and led to the loss of the internal symmetry of the $\mathrm{H}_{c}$ protons. However, different from the remarkably chemical shift ( $\beta$-CAR) before and after the complexation, the chemical shift of Chl-b changed slightly (Supplementary Figs 11-13). Furthermore, as shown in Supplementary Fig. 14a-d, according to the fluorescence intensity change of Chl-b (monitored at $450 \mathrm{~nm}$ ), the $K_{\mathrm{a}}$ of WP5 $\supset$ Chl-b (WCB) was calculated to be $(3.46 \pm 0.14) \times 10^{3} \mathrm{M}^{-1}$ (Supplementary Fig. 14e). The above results indicate that although Chl-b could thread into the cavity, the size mismatch between alkyl and cavity facilitates the alkyl in protruding from the cavity freely and thus could not result in the occupation of the cavity. The possible aggregates based on the unstable complexation was investigated, and no notable hierarchical nanostructure was observed when Chl-b was added to the solution of WP5 by using a similar method for HMS construction (Supplementary Fig. 15). Thus, based on the different binding affinities between WP5 $\supset \beta-C A R$ and WCB in binary solvents, Chl-b could play a role as a co-assembly component instead of a competitive guest during LHCs-b construction.

The route to synthesize the light-harvesting antenna is proposed in Fig. 4a. In the initial stage (step 1), the WCC is formed via host-guest recognition. According to the ${ }^{1} \mathrm{H}-\mathrm{NMR}$ results, WP5 is incapable of fixing Chl-b into a firm and durable cavity. Thus, once the cavity was filled by $\beta$-CAR, Chl-b had no opportunity to form complexation with WP5. In contrast, $\beta$-CAR could form complexation with WP5 when Chl-b protruded out of the cavity. Thus, WCC and Chl-b act as the main assembly factors. In this case, hydrophobic interactions further drive the WCC and Chl-b to assemble into LHCs containing Chl-b, in which the hydrophobic $\beta$-CAR and Chl-b tail are shielded from the water, whereas the hydrophilic WP5s and porphyrin are in contact with the aqueous environment (step 2). As in the LHCs containing Chl-b synthesis method, the $\beta$-CAR and Chl-b powders were first dissolved in ethanol, and then, $2 \mathrm{ml}$ of the 

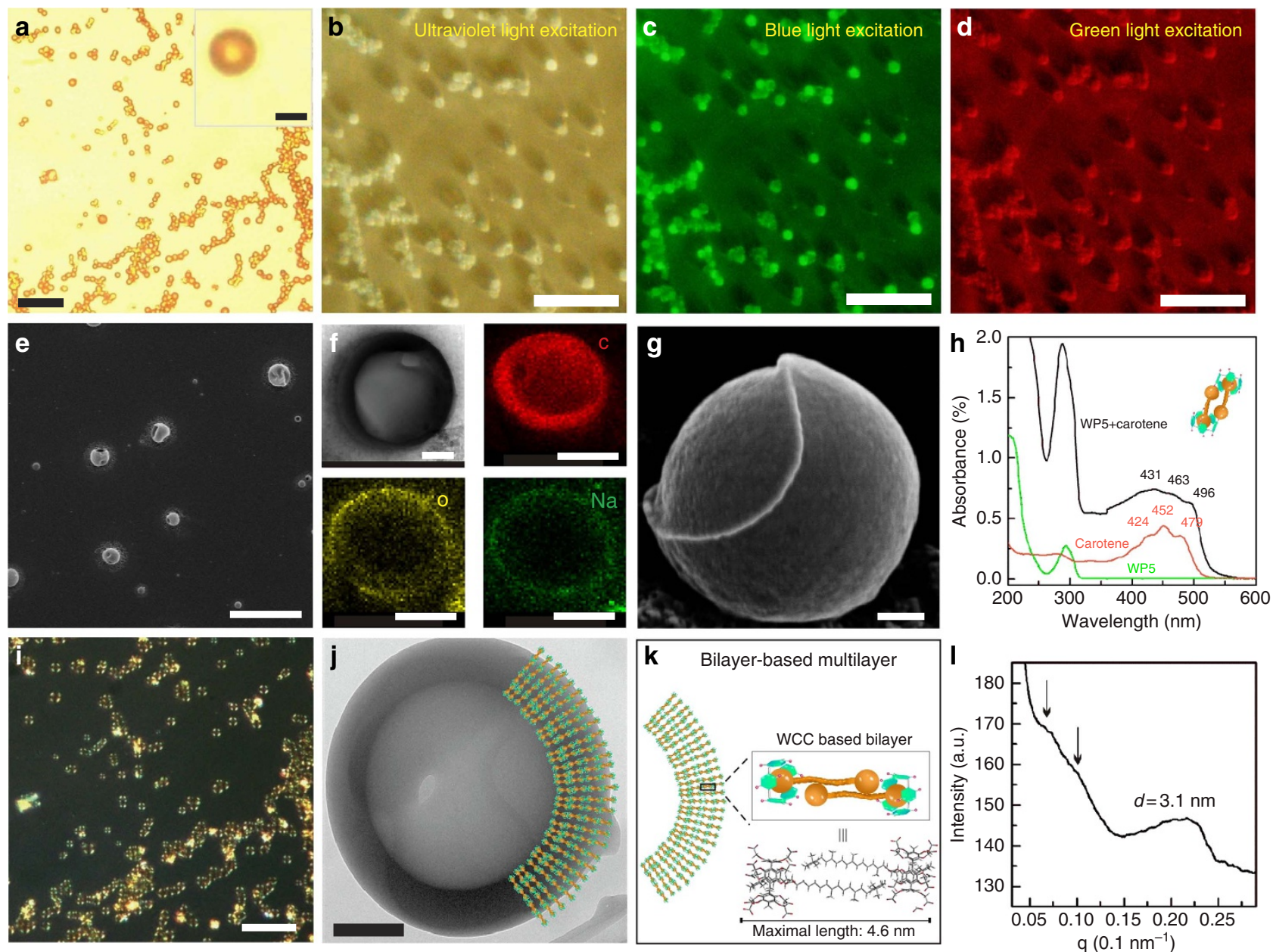

m

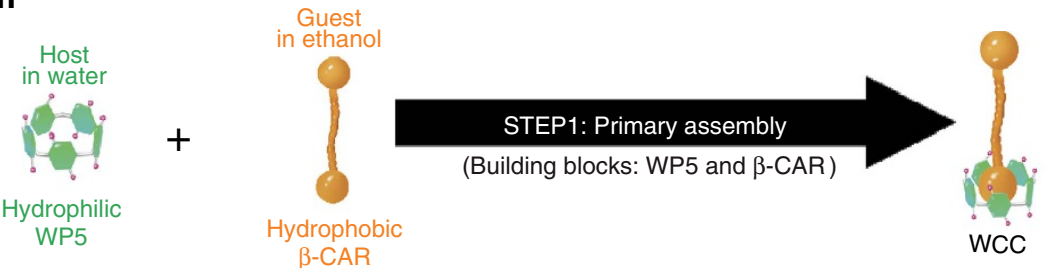

STEP2: Second-level assembly

(Building blocks: WCC)

WP5

B-CAR
B-CAdrophobic

WCC
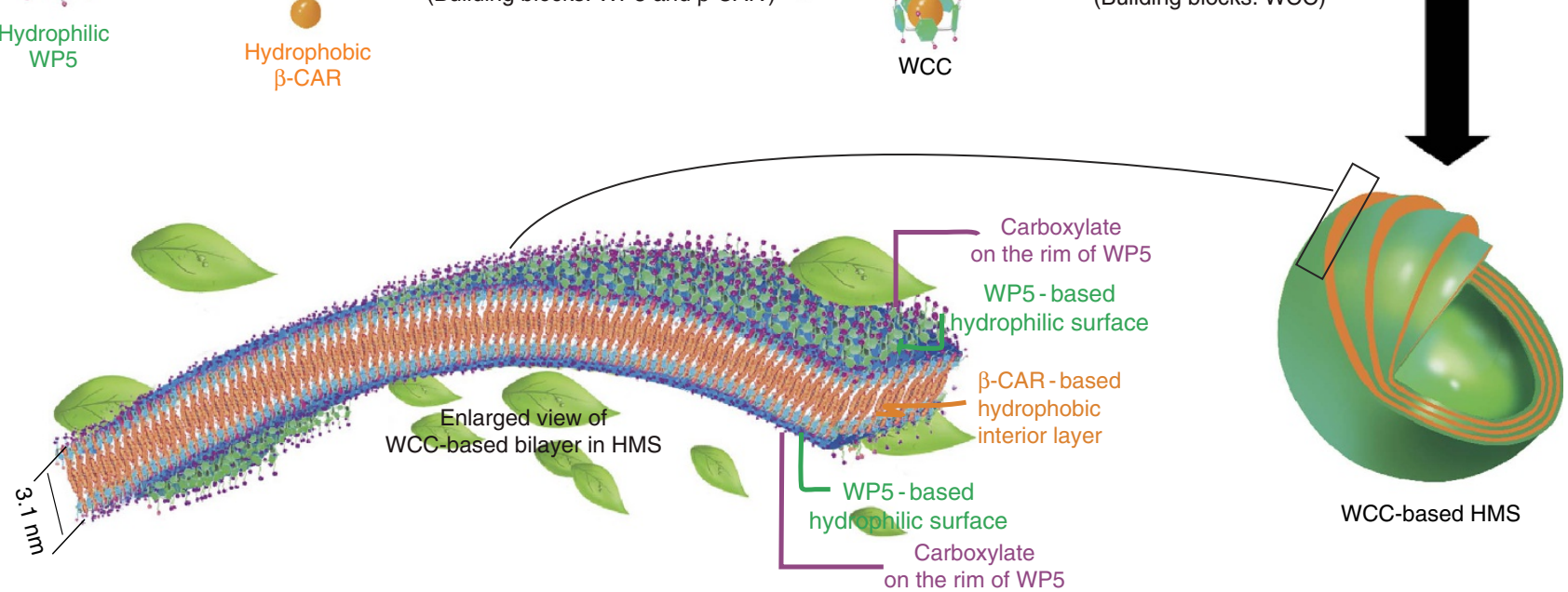

Figure 3 | Synthesis of WCC-based HMS. (a) OM image of HMSs over a large area. Scale bar, $10 \mu \mathrm{m}$ (inset: enlarged image of an HMS; Scale bar, $1 \mu \mathrm{m}$ ). Large-area fluorescence microscopy images of HMSs under (b) Ultraviolet-light excitation, (c) blue-light excitation and (d) green-light excitation. (e) SEM image of HMSs. Scale bar, $10 \mu \mathrm{m}$. (f) Top left, TEM image of an enlarged WCC-based HMS; Scale bar, 500 nm. EDX mapping images of a WCC-based HMS: top right, distribution of element C; lower left, distribution of element O; lower right, distribution of element Na. Scale bar, $1 \mu \mathrm{m}$. (g) SEM image of an HMS with a broken shell. Scale bar, $200 \mathrm{~nm}$. (h) ultraviolet-visible spectra of HMSs, $\beta$-CAR and WP5. (i) Polarized image of HMSs (the colour in this image is false). Scale bar, $10 \mu \mathrm{m}$. (j) TEM image of an HMS. Scale bar, $200 \mathrm{~nm}$. (k) Cartoon of WCC-based multilayers in the HMSs. (I) SAXS profile of the HMS sample (the final concentration of both HMS and $\beta$-CAR is $75 \mu \mathrm{M}$ ) with an acquisition time of $5 \mathrm{~h}$. (m) Schematic of the HMS formation process. 
a

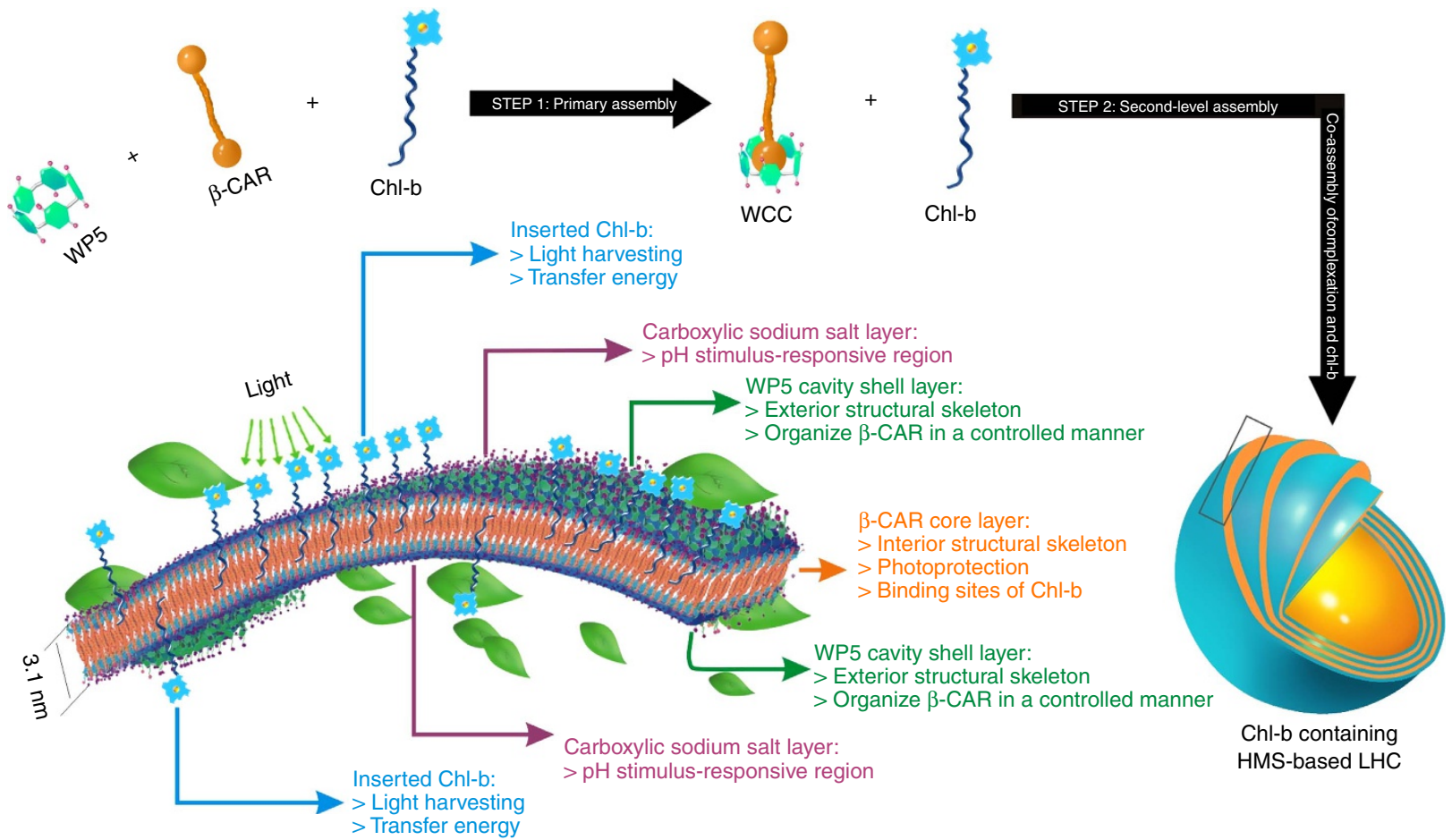

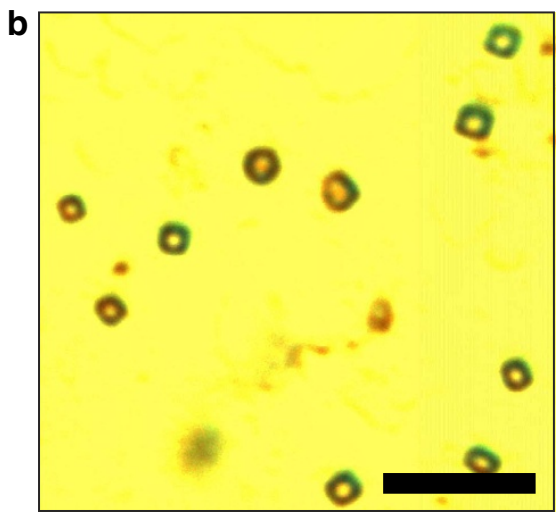

C
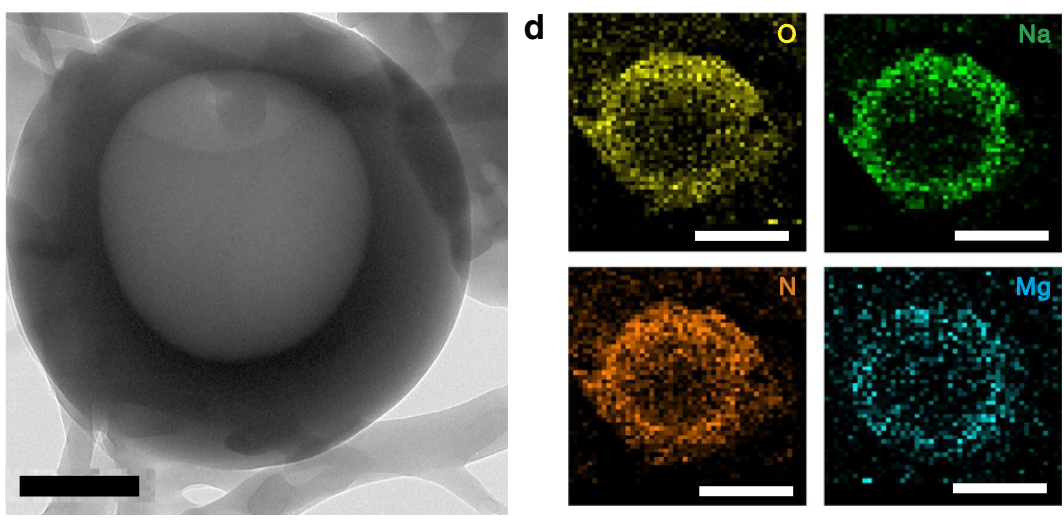

$\mathbf{e}$
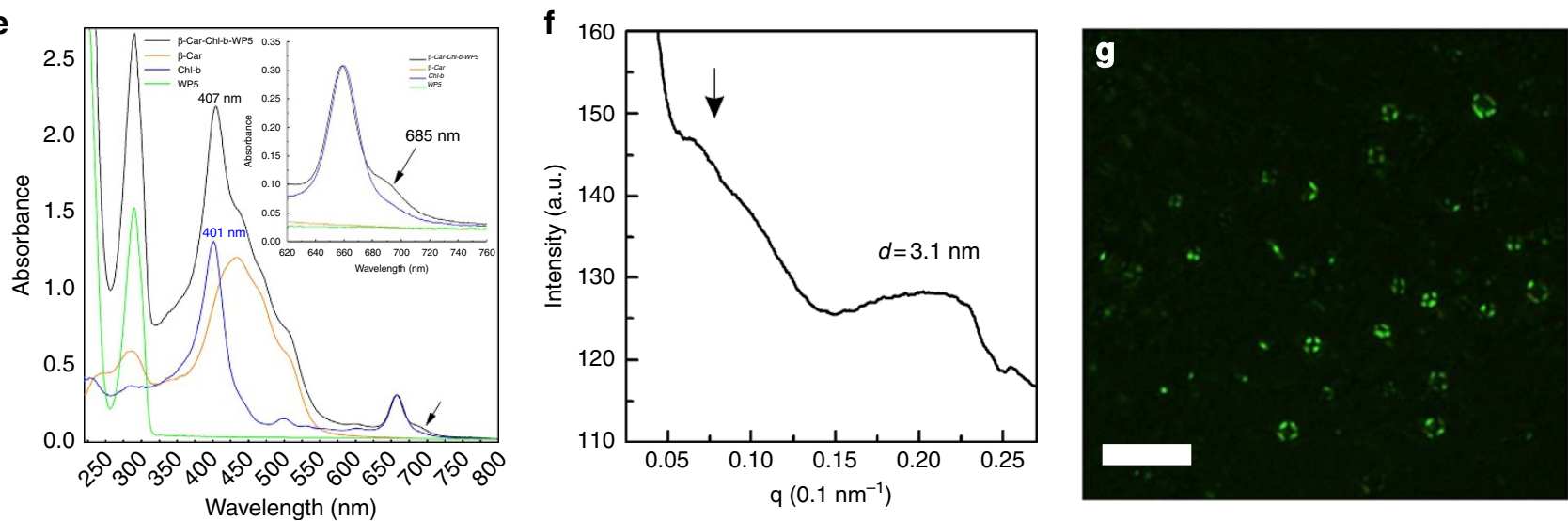

Figure 4 | Synthesis of HMS-based LHCs-b. (a) Synthesis of LHCs-b. (b) OM image showing LHCs-b. Scale bar, $10 \mu \mathrm{m}$. (c) TEM image of an LHCs-b. Scale bar, $200 \mathrm{~nm}$. (d) EDX mapping image of an LHCs-b (the colour in this image is false). Scale bar, $500 \mathrm{~nm}$. (e) Ultraviolet-visible spectra of $\beta$-CAR, Chl-b, WP5 and LHCs-b. (f) SAXS profile of an LHCs-b. (g) Polarized image of LHCs-b (the colour in this image is false). Scale bar, $10 \mu \mathrm{m}$.

$\beta$-CAR and $2 \mathrm{ml}$ of the Chl-b solutions were added to $4 \mathrm{ml}$ of an aqueous solution of WP5 using a pipette, without stirring. After the solution was aged for 7 days, pale-orange aggregates appeared.
Meanwhile, a clear Tyndall effect (Supplementary Fig. 16a) could be observed, indicating the formation of microaggregates. As shown in Fig. 4b, HMS-based LHCs containing Chl-b (LHCs-b) 
with orange-green-coloured walls were observed, confirming the homogeneous distribution of $\beta$-CAR and Chl-b throughout the LHCs-b. Compared with the narrow size distribution of HMSs (diameter range from 650 to $2,000 \mathrm{~nm}$ ), the DLS results showed that the insertion of Chl-b causes the size distribution to become wider (Supplementary Fig. 16b). OM observation demonstrated that both LHCs-b with diameters $<650 \mathrm{~nm}$ and $>2,000 \mathrm{~nm}$ co-existed and also possessed hollow spherical structures (Supplementary Fig. 17). It is worth noting that some green liquid could be found in the cavities of LHCs-b through OM observation (blue arrows). It is speculated that a small amount of Chl-b did not participate in the wall construction and, as a result, was encapsulated in the cavities of the hollow spheres during the formation of the hollow spheres. Chl-b solution shows no photocatalytic activity and they were isolated by the cavities. Thus, no further experiment was designed to remove the non-aggregated Chl-b. The TEM image in Fig. 4c (Supplementary Figs 18,19) demonstrates that the hollow spherical structure was maintained after the insertion of Chl-b. The elemental mapping analysis demonstrates the homogeneous distribution of $\mathrm{O}$ and $\mathrm{Na}$ (the characteristic elements in the WP5), as well as the homogeneous distribution of $\mathrm{N}$ and $\mathrm{Mg}$ (the characteristic elements in Chl-b), across the entire LHC (Fig. 4d, Supplementary Fig. 19). The ultraviolet-visible spectra of LHCs-b showed a red-shifted shoulder (at $685 \mathrm{~nm}$ ) absorption, which arose from alterations in the pigment-pigment interactions in LHCs-b (Fig. 4e). ${ }^{53}$ In addition, visible CD spectroscopy is a sensitive technique to monitor excitonic pigment-pigment and pigment-protein interactions ${ }^{54}$. As shown in Supplementary Fig. 20, the CD bands at $(-) 474,(-) 488,(+) 499,(-) 653$, $(-) 666,(+) 702$ and $(+) 724 \mathrm{~nm}$ were observed in LHCs-b. In the $\mathrm{Q}$ region, the $\mathrm{CD}$ spectra of the LHCs-b showed a negative peaks at $(-) 666 \mathrm{~nm}$, which is characteristic of Chl-b ${ }^{55}$ and accompanied by negative peak at $(-) 653 \mathrm{~nm}$. The positive peaks at $(+) 499 \mathrm{~nm}$ and $(-) 474 \mathrm{~nm}$ in the CD band were accompanied by a negative peak at $(-) 488 \mathrm{~nm}$, originating from $\beta-\mathrm{CAR}^{56}$. Notably, the band at $649 \mathrm{~nm}$ (Chl-b in ethanol) was red-shifted to $666 \mathrm{~nm}$ with a shoulder at $653 \mathrm{~nm}$ on constitution into HMS. These results indicate that the Chl-b-Chl-b and/or Chl-b- $\beta$-CAR interactions were affected by constituting into HMS, which resulted from the changes in microenvironment of Chl-b in LHCs- $b^{57}$. The bands at $(-) 474$ and $(+) 499 \mathrm{~nm}$ as well as those at $(-) 666 \mathrm{~nm}$ and $(+) 724 \mathrm{~nm}$ may originate from excitonic interactions involving Chl-b and $\beta$-CAR molecules. The integrated areas of the positive and negative bands appear to be approximately equal ${ }^{58}$. These spectra suggest that in LHCs-b, a complicated set of excitonic interactions occurs between several Chl-b and $\beta$-CAR molecules ${ }^{59}$. Furthermore, the peak obtained from SAXS showed that the d-spacing remains at $3.1 \mathrm{~nm}$ (Fig. 4f), which provides further evidence that the ordered structure of the HMS was not destroyed after the co-assembly with Chl-b. In addition, the strong anisotropic photoluminescence shown in Fig. $4 \mathrm{~g}$ is in good agreement with the SAXS data. Because the $K_{\mathrm{a}}$ of WP5 $\supset \beta$-CAR was higher than that of WCB by two orders of magnitude, it could be deduced that almost all of the $\beta$-CAR in the system was wrapped by WP5. Thus, the position of $\beta$-CAR mainly located in the wall of the LHCs-b. Considering that hierarchical aggregates could not be obtained by WCB, a large amount of Chl-b, but not WCB randomly inserted into the HMS due to non-specific interactions. Therefore, it is hard to obtain a constant proportion of Chl-b among all LHCs-b (caused by the randomly insertion manner). As a result, it is difficult to obtain a convincing data by Elementary Analysis or Inductive Coupled Plasma Emission Spectrometer (proportion of Chl-b in each LHCs-b is different). Fortunately, EDX spectra provide a fine-testing method to investigate the $\beta$-CAR/Chl-b ratio in individual LHC-b. Thus, as shown in Supplementary Fig. 19a-d, LHCs-b with different diameter were selected and analysed. On the basis of the obtained semi-quantitative data, rough range of the Chl-b proportion could be provided (Supplementary Fig. $19 \mathrm{a}-\mathrm{d}$ inset). Then, the average $\beta$-CAR/Chl-b ratio of $\sim 1.8$ was obtained by calculation. Being confined to the accuracy of the semi-quantitative analysis (and interference of Chl-b solution in cavities), these data are provided only for providing a rough estimate.

HMS-based LHCs served as photocatalytic entities. The catalytic activity of LHCs-b was evaluated for the reduction of the pollutant 4-nitrophenol (4-NP) after the addition of $\mathrm{NaNO}_{2}$. Figure $5 \mathrm{a}$ shows the ultraviolet-visible spectra of 4 -NP in ethanol/water solutions containing distilled water (blank sample), $\beta$-CAR, Chl-b, HMS and LHCs-b; in this case, the photoirradiation time was $0 \mathrm{~min}$. All of these spectra display a main absorption at $318 \mathrm{~nm}$ (4-NP) and a shoulder peak at $400 \mathrm{~nm}$ (4-nitrophenolate ions). After irradiation with a mercury lamp $(500 \mathrm{~W})$ for $10 \mathrm{~min}$ (Fig. $5 \mathrm{~b}$ ), the peak intensity at $318 \mathrm{~nm}$ decreased and the peak intensity at $400 \mathrm{~nm}$ increased for all of the samples due to the formation of 4-nitrophenolate ions ${ }^{60}$. Importantly, a new peak at $290 \mathrm{~nm}$ was observed in the spectrum of the sample of LHCs-b (blue line), which indicated the generation of 4-aminophenol (4-AP) and further demonstrated the photocatalytic activity of LHCs-b. Remarkably, after being irradiated with the mercury lamp for $20 \mathrm{~min}$, the HMS sample also exhibited photocatalytic activity towards 4-NP; as shown in Fig. 5c, a new peak at $290 \mathrm{~nm}$ appeared in the HMS spectrum (green line).

These findings reveal that $\beta$-CAR suprastructures also exhibited catalytic activity. Reasonably, as the irradiation time was prolonged (Fig. 5d), a peak was also observed at $290 \mathrm{~nm}$ in the spectra of HMS and LHCs-b. As revealed by the literature, the carotene molecules form a layer between $0-40-\mathrm{mm}$ deep under the insect's cuticle, putting them in the perfect position to capture the Sun's light. Similar to the literature, the hierarchical way in which the carotene molecules are arranged in our studies provides the possibility of photocatalytic activity by the HMS ${ }^{61,62}$. Control experiments using untreated samples were performed for the same samples at different time intervals in the absence of photoirradiation. As shown in Fig. 5e-h, no changes were observed in the ultraviolet-visible spectra compared with those of the experimental group (Fig. 5a-d). Subsequently, LHCs-b were irradiated for up to $120 \mathrm{~min}$ (in $5 \mathrm{~min}$ intervals, from $0-120 \mathrm{~min}$ ). As shown in Fig. 5i, when a mixture of 4-NP and LHCs-b was irradiated, the intensity of the peak at $290 \mathrm{~nm}$ increased due to the generation of 4-AP, which further confirmed that the LHCs-b can be used as a photocatalyst for the reduction of organic species.

Excitingly, when an aqueous solution of $\mathrm{K}_{2} \mathrm{PtCl}_{4}$, ascorbic acid (as an electron donor) and LHCs-b was irradiated for $2 \mathrm{~h}$, LHC microspheres decorated with platinum nanoparticles were observed (Fig. 5j). Similarly, photoreduction of an $\mathrm{Ag}^{\mathrm{I}}$ salt occurred within $2 \mathrm{~h}$ of irradiation and produced AgNP-loaded spheres, as revealed by TEM (Supplementary Fig. 21). Palladium nanoparticles were similarly obtained (Fig. 5k). The photocatalytic reaction is believed to occur through two steps. First, Chl-b captures light to produce photoexcited states that are rapidly reduced by an electron donor. Then, the resulting Chl-b radical anion can be used as a catalyst to reduce various metal salts to their metallic state through successive light-harvesting and photochemical cycles (Fig. 51) ${ }^{63}$. Microspheres with stimuli responsiveness are extremely important for potential applications. A decrease in $\mathrm{pH}$ induces marked damage in HMSs (Fig. 5m), 
a

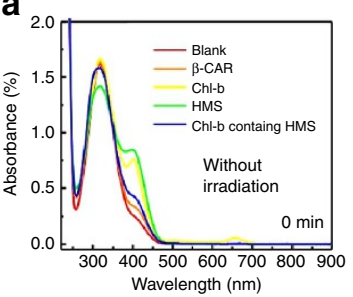

e

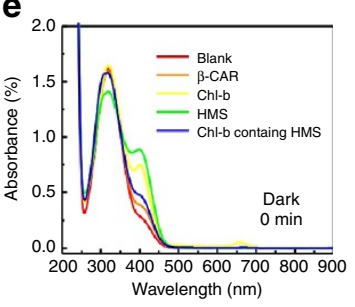

b
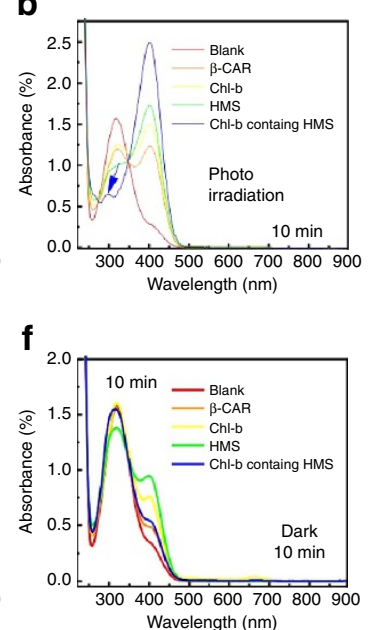

C

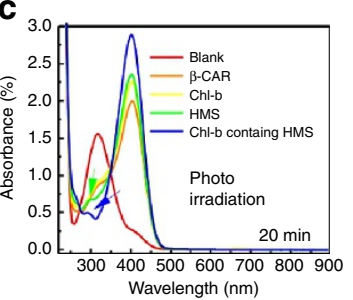

g

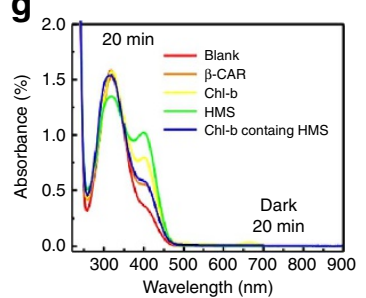

d

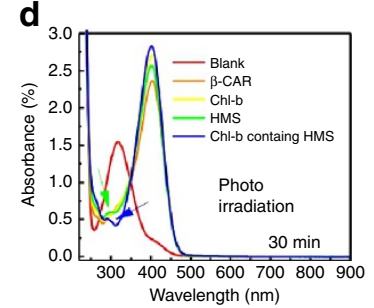

h

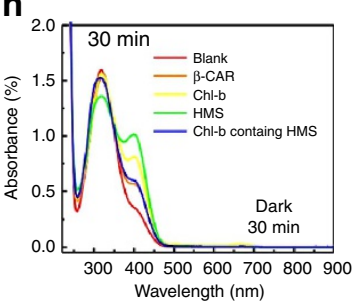

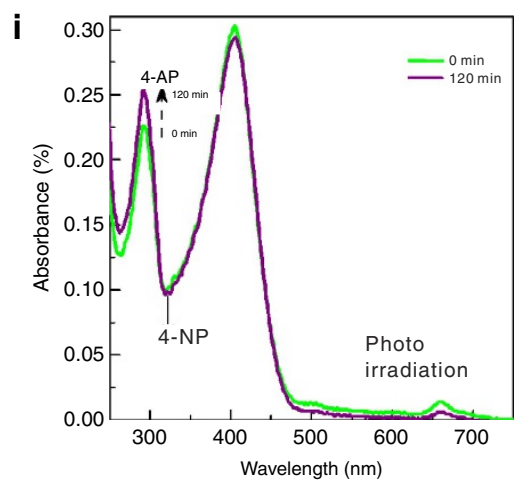
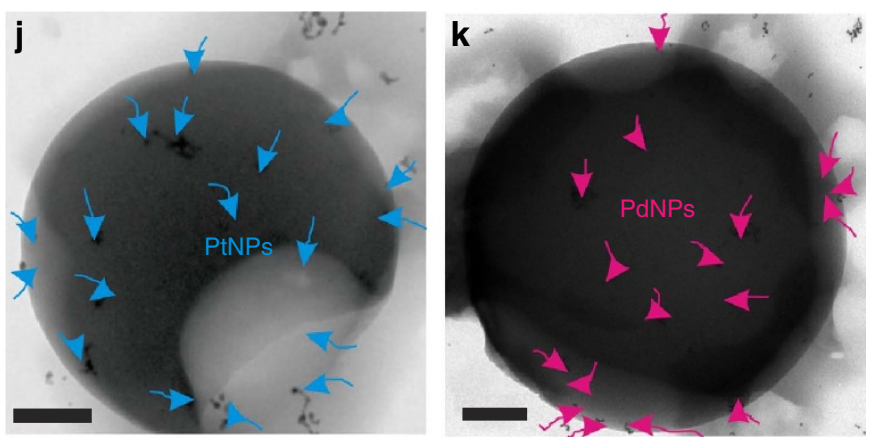

\section{I}

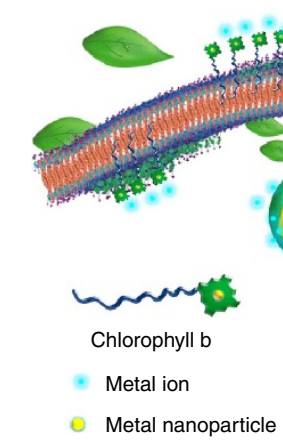

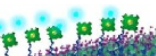
ax

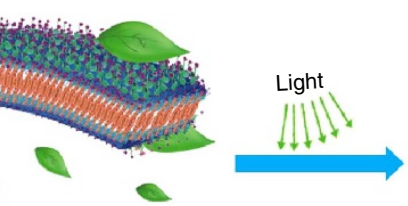

(2):

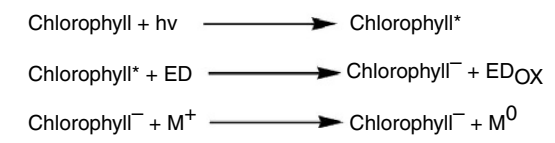

Chlorophyll
Chlorophyll
$\mathrm{ED}_{\text {ox }}$
$\mathrm{M}^{+}$and $\mathrm{M}^{0}$

Photoexcited state of the chlorophyll;

Chlorophyll radical ion;
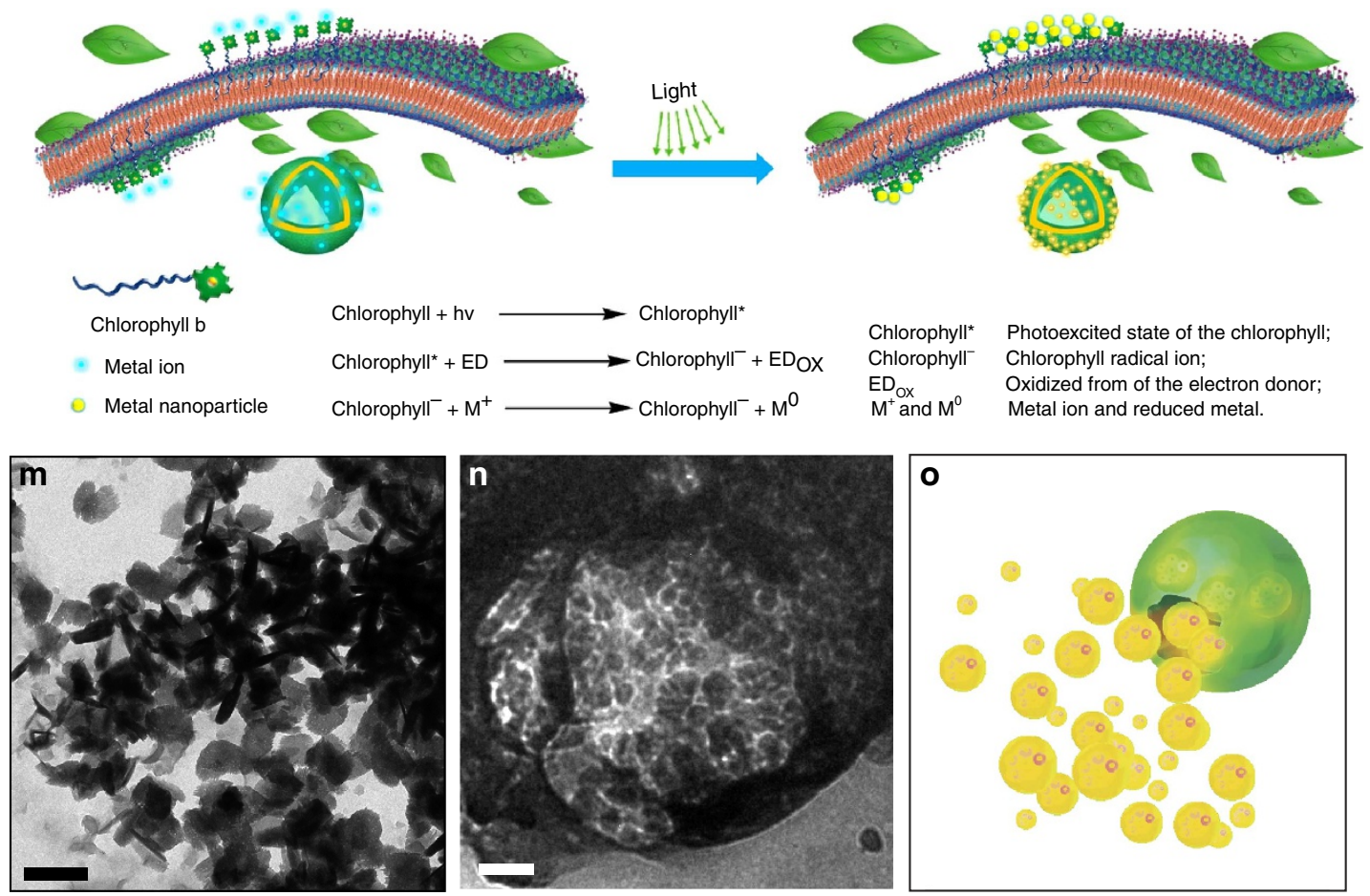

Figure 5 | HMS-based LHCs-b served as photocatalytic entities. Ultraviolet-visible spectra of the transformation of 4-NP into 4-AP under photocatalysis by $\beta$-CAR, Chl-b, HMS, and LHCs-b for (a) $0 \mathrm{~min},(\mathbf{b}) 10 \mathrm{~min}$, (c) $20 \mathrm{~min}$ and (d) $30 \mathrm{~min}$. (e-h) are the corresponding untreated control groups for (a-d). (i) Ultraviolet-visible spectra of the transformation of 4-NP into 4-AP under photocatalysis by LHCs-b. TEM images of $\mathbf{j}$, a PtNPs-loaded LHCs-b (the blue arrows indicate the PtNPs); Scale bar, $100 \mathrm{~nm}$, and (k) a PdNPs-loaded LHCs-b (the pink arrows indicate the PdNPs); Scale bar, $200 \mathrm{~nm}$. (I) Possible photosynthesis mechanism of LHCs-b. (m) TEM micrograph of a self-broken HMS during the HCl-triggered degradation. Scale bar, $500 \mathrm{~nm}$. (n) TEM micrograph of an HMS containing AgNPs during the $\mathrm{HCl}$-triggered degradation. Scale bar, $100 \mathrm{~nm}$. (o) Schematic showing the release of nanoparticles. 
which appears to be attributable to a transition from a microspherical structure to a micellar structure. Therefore, the loading and release of guest molecules in response to a decrease in $\mathrm{pH}$ was investigated. Here, WP5-functionalized AgNPs used as a model guest were encapsulated in the microsphere interior during assembly. When the solution $\mathrm{pH}$ was adjusted to produce acidic conditions, the release of AgNPs from the cavity of an HMS was clearly observed (Fig. 5n). Figure 5o shows a schematic of the release of loaded nanoparticles, demonstrating the possible application of this system for controlled release.

\section{Discussion}

In previous reports of $\beta$-CAR with host $\beta$-cyclodextrin or calixarenes, $\beta$-cyclodextrin was used to encapsulate the bulky group of $\beta$-CAR ${ }^{64,65}$ and calixarenes were used as nanocontainers. The natural $\beta$-cyclodextrins are concave macrocycles capable of expressing molecular recognition via the formation of noncovalent inclusions with suitably sized hydrophobic guests in their cavities. These complexes are currently used in many applications, including enhancing the water solubility of hydrophobic guests, controlling the release of volatile guests and protecting labile guests from degradation promoted by external agents. As for the calixarenes, $\beta$-CAR molecules were simply loaded in the hydrophilic vector by spinning disk processing. In this sense, a study of the association between $\beta$-CAR and other hosts is very important because it could provide better binding sites for carotenoids, allowing improved structural control via higher binding specificity (Supplementary Discussion). The formation of the WCC was supported by the Raman and ${ }^{1} \mathrm{H}-\mathrm{NMR}$ spectra, which demonstrated that the binding site was in the terpene section of $\beta$-CAR based on the hydrophobic interactions. The generation of an HMS must be associated with the structural characteristics of the WCC; when either WP5 or $\beta$-CAR was removed from the water or ethanol, no HMS formation was observed (Supplementary Fig. 22), convincingly demonstrating that the WCC formed by WP5 and $\beta$-CAR is undoubtedly the critical factor for the construction of HMSs. Interestingly, HMSs can provide a better chemical system in terms of stability without the loss of bioavailability (Supplementary Figs 23,24). The HMS was observed to display characteristics that differ from those of traditional synthetic molecule-based suprastructures (Supplementary Fig. 25). Furthermore, the peak in the SAXS profile is weak as a consequence of the low concentration of HMSs. As shown in Supplementary Fig. 26, the intensity of the peak increased with increasing acquisition time.

As shown in Supplementary Fig. 27, LHC containing Chl-a could also be obtained based on WCC-based HMS. LHCs-b exhibit photocatalytic activity, as was demonstrated through a series of experiments. Interestingly, the HMSs also exhibit photoactivity, which differs from that of LHCs-b in that it exhibits slower kinetics.

The successful creation of HMS provides sophisticated strategies to generate carotenoids-based hierarchical model, which will result in innovative approaches to generate controllable supramolecular structures composed of carotenoids. The introduction of various biomolecules, could lead to the development of this supramolecular platform into diverse artificial biological cells for mimicking and optimizing photosynthetic systems, which would further provide a photosynthetic model and important tools for the investigation of the origins of the bioenergy system in living organisms.

\section{Methods}

General considerations. All chemical reagents were purchased from Aladdin and used without further purification. ${ }^{1} \mathrm{H}$-NMR spectra were recorded on a Bruker
Advance $600 \mathrm{MHz}$ spectrometer using $\mathrm{D}_{2} \mathrm{O}$ as the solvent. $\beta$-CAR and Chl-b were purchased from EKEAR (Shanghai). Raman spectra were measured in solution using an In Renishaw Via Raman spectrometer $\left(\lambda_{\text {exc }}=532 \mathrm{~nm}\right)$. For Raman measurements, aqueous dispersions were dried onto glass slides; the focus was then centred on an individual HMS for detection. Fluorescence spectra were recorded on an F-4500 spectrometer; the spectra were corrected against a photomultiplier and against the lamp intensity. The slit width of both monochromators was $5.0 \mathrm{~nm}$. ultraviolet-visible absorption spectra of the photocatalytic experiment (the experimental group and the untreated control group) were recorded on a UV-2550 spectrophotometer. Ultraviolet-visible absorption spectra for assembly characterization and photocatalysis experiments $(0-120 \mathrm{~min})$ were recorded on a UV-2501 spectrophotometer. FT-IR spectra were collected using potassium bromide pellets on a TENSOR 27 spectrometer. Circular dichroism spectra were measured in a Jasco-815 spectropolarimeter. To compare the shift of each peak more clearly, the intensity of the $649 \mathrm{~nm}$ peak in the LHCs containing Chl-b was adjusted to the same intensity as the base of Chl-b. Dynamic light scattering and zeta-potential measurements were carried out on a Malvern Nanosizer S instrument at room temperature. $\mathrm{OM}$ and polarized $\mathrm{OM}$ images were obtained using a Leica Microsystems DM LM/P instrument. Fluorescence OM images were acquired on an Olympus IX 73 W. D. $27 \mathrm{~mm}$ with an inverted configuration. For $\mathrm{OM}$, polarized $\mathrm{OM}$ and fluorescence $\mathrm{OM}$, aqueous dispersions of orange aggregates were dried onto glass slides for observation. The TEM images were obtained using a Philips TECNAI-12 instrument with an accelerating voltage of $120 \mathrm{kV}$. For TEM, aqueous dispersions of HMSs and Chl-b-containing LHCs were dried onto carbon-coated copper support grids. A Hitachi S-4800 field-emission scanning electron microscope was used to investigate the HMS. For SEM, aqueous dispersions of HMSs were dried onto silicon wafers. HRTEM and elemental mapping images were obtained using a Tecnai G2 F30 S-TWIN instrument. SAXS experiments were performed at room temperature on a NanoSTAR, Bruker-AXS, $30 \mathrm{~W}$. For SAXS, dispersions of WCC-based HMSs and Chl-b-containing LHCs were loaded into special glass X-ray capillary tubes with an internal diameter of $1.5 \mathrm{~mm}$.

Synthesis of pillar[5]arene WP5. Carboxyl-modified pillar[5] arene WP5 was prepared by a modified literature procedure ${ }^{38}$ and was identified by ${ }^{1} \mathrm{H}-\mathrm{NMR}$ spectroscopy in $\mathrm{D}_{2} \mathrm{O} .{ }^{1} \mathrm{H}-\mathrm{NMR}\left(\mathrm{D}_{2} \mathrm{O}, 600 \mathrm{MHz}\right.$, p.p.m.): $\delta 6.7$ (s, $\left.10 \mathrm{H}\right), 4.28$ $(\mathrm{d}, 20 \mathrm{H}), 3.90(\mathrm{~s}, 10 \mathrm{H})$.

Characterization of chemical and biomolecular materials. $\beta$-CAR (purity $\geq 97 \%$ ) and Chl-b (purity $\geq 95 \%$ ) were purchased from EKEAR (Shanghai). WP5 was prepared by a modified literature procedure and was identified by ${ }^{1} \mathrm{H}-\mathrm{NMR}$ spectroscopy in $\mathrm{D}_{2} \mathrm{O}$

WCC studies. For ${ }^{1} \mathrm{H}-\mathrm{NMR}$ characterization, $\beta$-CAR was dissolved in ethanol- $\mathrm{d}^{6}$ to produce a $150 \mu \mathrm{M}$ solution (curve a in Supplementary Figs 1, 2, solvent: ethanol- $\mathrm{d}^{6}$ ). WP5 was dissolved in $\mathrm{D}_{2} \mathrm{O}$ to produce a $300 \mu \mathrm{M}$ aqueous solution, and then, an equal volume of ethanol- $\mathrm{d}^{6}$ was added to the WP5 solution to produce a $150 \mu \mathrm{M}$ binary solvent solution (curve $\mathrm{c}$ in Supplementary Figs 1, 2, solvent: ethanol- $\left.\mathrm{d}^{6}: \mathrm{D}_{2} \mathrm{O}=1: 1\right)$. The $\mathrm{D}_{2} \mathrm{O}$ aqueous solution of WP5 added to the ethanol- $\mathrm{d}^{6}$ solution to produce a $150 \mu \mathrm{M}$ WP5 and $\beta$-CAR complexation solution (curve b in Supplementary Figs 1, 2, solvent: ethanol- $\mathrm{d}^{6}: \mathrm{D}_{2} \mathrm{O}=1: 1$ ). Clear ${ }^{1} \mathrm{H}-\mathrm{NMR}$ spectra could not be achieved when $\beta$-CAR ethanol- $\mathrm{d}^{6}$ solution was added by an equal volume of $\mathrm{D}_{2} \mathrm{O}$ due to the poor solubility and low concentration of $\beta$-CAR in water in the absence of WP5. Thus, to obtain clear spectra, ethanol- $\mathrm{d}^{6}$ instead of binary solvents (ethanol- $\mathrm{d}^{6}: \mathrm{D}_{2} \mathrm{O}=1: 1$ ) were used for the $\beta$-CAR ${ }^{1} \mathrm{H}-\mathrm{NMR}$ characterization and for further comparison. In addition, the comparison between $\beta$-CAR (ethanol- $\mathrm{d}^{6}$ ) and WCC (ethanol- $\mathrm{d}^{6}: \mathrm{D}_{2} \mathrm{O}=1: 1$ ) is closer to the solvent environment changes in the assembly process conditions. For fluorescence characterization, various concentrations of $\beta$-CAR in ethanol solution were added to an aqueous solution of WP5 to produce $\beta$-CAR/WP5 solutions with different molar ratios of $0,0.2,0.3,0.6,0.8,1.2,1.6,2.0$ and 2.5 . The emission intensities of the WP5 solutions at various molar ratios were monitored by fluorescence spectroscopy $\left(\lambda_{\mathrm{ex}}=268 \mathrm{~nm}\right)$. Using a non-linear curve-fitting method, the $K_{\mathrm{a}}$ of WP5 $\supset \beta$-CAR was estimated.The non-linear curve fitting was based on the equation $\Delta F=\left(\Delta F_{\infty} /[\mathrm{H}]_{0}\right)\left(0.5[\mathrm{G}]_{0}+0.5\left([\mathrm{H}]_{0}+1 / K_{\mathrm{a}}\right)-\left(0.5\left([\mathrm{G}]_{0}^{2}+\right.\right.\right.$ $\left.\left.\left.\left(2[\mathrm{G}]_{0}\left(1 / K_{\mathrm{a}}-[\mathrm{H}]_{0}\right)\right)+\left(1 / K_{\mathrm{a}}+[\mathrm{H}]_{0}\right)^{2}\right)^{0.5}\right)\right)(1)($ refs 37,66$)$. On addition of $\beta$-CAR, the fluorescence intensity of WP5 (monitored at $653 \mathrm{~nm}$ ) was gradually quenched, and the association constant of WP5 $\supset \beta$-CAR was calculated. $\Delta F$ is the fluorescence intensity changes at $653 \mathrm{~nm}$ at $[\mathrm{G}]_{0} ; \Delta F_{\infty}$ is the fluorescence intensity changes at $653 \mathrm{~nm}$ when WP5 is completely complexed, $[\mathrm{G}]_{0}$ is the initial concentration of $\beta-\mathrm{CAR},[\mathrm{H}]_{0}$ is the fixed initial concentration of WP5.

WP5 and Chl-b interaction studies. For ${ }^{1} \mathrm{H}-\mathrm{NMR}$ characterization, Chl-b was dissolved in ethanol- $\mathrm{d}^{6}$ to produce a $150 \mu \mathrm{M}$ solution (curve a in Supplementary Figs 11-13, solvent: ethanol- $\mathrm{d}^{6}$ ). WP5 was dissolved in $\mathrm{D}_{2} \mathrm{O}$ to produce a $300 \mu \mathrm{M}$ aqueous solution, and then, an equal volume of ethanol- $\mathrm{d}^{6}$ was added to WP5 solution to produce a $150 \mu \mathrm{M}$ binary solvent solution (curve $\mathrm{c}$ in Supplementary Figs 11-13, solvent: ethanol- $\left.\mathrm{d}^{6}: \mathrm{D}_{2} \mathrm{O}=1: 1\right)$. The $\mathrm{D}_{2} \mathrm{O}$ aqueous solution of WP5 
added to the ethanol- $\mathrm{d}^{6}$ solution to produce a $150 \mu \mathrm{M}$ WP5 and Chl-b complexation solution (curve b in Supplementary Figs 11-13, solvent: ethanol- $\mathrm{d}^{6}$ : $\left.\mathrm{D}_{2} \mathrm{O}=1: 1\right)$. In accordance with the method used in previous experiments (WCC ${ }^{1} \mathrm{H}-\mathrm{NMR}$ characterization), ethanol-d ${ }^{6}$ was used for the Chl-b ${ }^{1} \mathrm{H}-\mathrm{NMR}$ characterization and for further comparison instead of binary solvents (ethanol- $\mathrm{d}^{6}$ : $\left.\mathrm{D}_{2} \mathrm{O}=1: 1\right)$

To determine the $K_{\mathrm{a}}$ for the complexation between WP5 and Chl-b, fluorescence titration experiments were carried out in solutions that had a constant concentration of Chl-b and varying concentrations of WP5. Various concentrations of WP5 solutions were added to an ethanol solution of Chl-b at the concentration of $100 \mu \mathrm{M}$ $(1,000 \mu \mathrm{l})$ to produce WP5/Chl-b solutions with different molar ratios $(0,0.2,0.4$, $0.6,0.8,1.0,1.2,1.4,1.6,2.2,2.6$ and 2.8). In a typical experiment, $1,000 \mu$ lo WP5 at the concentration of $20 \mu \mathrm{M}$ were added to the Chl-b solution to produce WP5/Chl-b solutions with a molar fraction of 0.2 . Then, $1,000 \mu \mathrm{l}$ of WP5 at the concentration of $40 \mu \mathrm{M}$ were added to the Chl-b solution to produce WP5/Chl-b solutions with a molar fraction of 0.4. Subsequently, 1,000 $\mu \mathrm{l}$ of WP5 at the concentration of $60 \mu \mathrm{M}$ were added to the Chl-b solution to produce WP5/Chl-b solutions with a molar fraction of 0.6 . The solutions with the other molar ratios $(0.8,1.0,1.2,1.4,1.6,2.2,2.6$ and 2.8) were prepared by a similar method. The emission intensities of the WP5 solutions at various molar ratios were monitored by fluorescence spectroscopy $\left(\lambda_{\mathrm{ex}}=268 \mathrm{~nm}\right)$. The non-linear curve fitting was based on the equation $\Delta F=$ $\left(\Delta F_{\infty} /[\mathrm{G}]_{0}\right)\left(0.5[\mathrm{H}]_{0}+0.5\left([\mathrm{G}]_{0}+1 / \mathrm{Ka}\right)-\left(0.5\left([\mathrm{H}]_{0}^{2}+\left(2[\mathrm{H}]_{0}\left(1 / K_{\mathrm{a}}-[\mathrm{G}]_{0}\right)\right)+(1 /\right.\right.\right.$ $\left.\left.\left.\left.K_{\mathrm{a}}+[\mathrm{G}]_{0}\right)^{2}\right)^{0.5}\right)\right)(2)($ refs 37,66), which is was obtained by a modified literature equation (fix the concentration of guest and increase the concentration of host) According to the different emission peaks of WP5 and Chl-b in the range from 300 to $750 \mathrm{~nm}$, the peak at $450 \mathrm{~nm}$ was selected to monitor the intensity change of Chl-b. On the basis of the fluorescence intensity change of Chl-b (monitored at $450 \mathrm{~nm}$ ), the association constant of WCB was calculated. $\triangle F$ is the fluorescence intensity change at $450 \mathrm{~nm}$ at $[\mathrm{H}]_{0}, \Delta F_{\infty}$ is the fluorescence intensity change at $450 \mathrm{~nm}$ when WP5 is completely complexed; $[\mathrm{G}]_{0}$ is the fixed initial concentration of Chl-b; $[\mathrm{H}]_{0}$ is the initial concentration of WP5.

Synthesis of HMSs. WP5 was first basified with sodium hydroxide to produce an aqueous solution. Then, $\beta$-CAR was dissolved in ethanol to produce an ethanol solution of $\beta$-CAR. Subsequently, $\beta$-CAR-based HMSs were prepared by pipetting an ethanol solution of $\beta$-CAR into an aqueous solution of WP5 and then aging. The final concentration of host and guest could be controlled by adjusting their initial concentration. In a typical experiment, HMSs were prepared by pipetting $2 \mathrm{ml}$ of an ethanol solution of $\beta$-CAR $(150 \mu \mathrm{M})$ into $2 \mathrm{ml}$ of an aqueous solution of WP5 $(150 \mu \mathrm{M})$ and then aging the solution for 7 days at room temperature.

Synthesis of LHCs-b. WP5 was first basified with sodium hydroxide to produce an aqueous solution. Then, $\beta$-CAR was dissolved in ethanol to produce an ethanol solution of $\beta$-CAR. Chl-b was dissolved in ethanol to produce an ethanol solution of Chl-b. Simlar to the synthesis method of HMSs, LHCs-b were prepared at room temperature by pipetting an ethanol solution of $\beta$-CAR and Chl-b into an aqueous solution of WP5. The final concentration of host and guest could be controlled by adjusting their initial concentration. In a typical experiment, LHCs-b were prepared at room temperature by pipetting $2 \mathrm{ml}$ of an ethanol solution of $\beta$-CAR $(150 \mu \mathrm{M})$ and $2 \mathrm{ml}$ of an ethanol solution of Chl-b $(150 \mu \mathrm{M})$ into $4 \mathrm{ml}$ of an aqueous solution of WP5. Mixing the solution resulted in a pale-orange turbid suspension that was subsequently aged for 7 days.

Photocatalytic synthesis of metal nanoparticles. In a typical experiment, $0.50 \mathrm{ml}$ of a suspension of LHCs-b, $20 \mu \mathrm{l}$ of aqueous $\mathrm{K}_{2} \mathrm{PtCl}_{4}(10 \mathrm{mM})$ and $25 \mu \mathrm{l}$ of aqueous ascorbic acid $(0.2 \mathrm{M})$ were sequentially added to a 2 -ml glass vial and then irradiated for $2 \mathrm{~h}$ with visible light from a 300-W Xe lamp. Pd and Ag nanoparticles were prepared using similar methods.

Photocatalytic reduction of 4-NP. Experimental group. Blank sample: $1 \mathrm{ml}$ of 4NP stock solution $(2 \mathrm{mM}), 100 \mu \mathrm{l}$ of sodium nitrite stock solution $(0.1 \mathrm{M})$ and $300 \mu$ lof distilled water were mixed and homogenized in a glass vial and then irradiated with visible light from a 500-W mercury lamp. $\beta$-CAR sample: One millilitre of 4-NP stock solution $(2 \mathrm{mM}), 100 \mu \mathrm{l}$ of sodium nitrite stock solution $(0.1 \mathrm{M})$ and $300 \mu \mathrm{l}$ of a dispersion of $\beta$-CAR $(75 \mu \mathrm{M})$ were mixed and homogenized in a glass vial and then irradiated with visible light from a $500-\mathrm{W}$ mercury lamp. Chl-b sample: $1 \mathrm{ml}$ of 4 -NP stock solution $(2 \mathrm{mM}), 100 \mu \mathrm{l}$ of sodium nitrite stock solution $(0.1 \mathrm{M})$ and $300 \mu \mathrm{l}$ of a dispersion of Chl-b $(75 \mu \mathrm{M})$ were mixed and homogenized in a glass vial and then irradiated with visible light from a $500-\mathrm{W}$ mercury lamp. HMS sample: $1 \mathrm{ml}$ of 4 -NP stock solution $(2 \mathrm{mM}), 100 \mu \mathrm{l}$ of sodium nitrite stock solution $(0.1 \mathrm{M})$ and $300 \mu \mathrm{l}$ of a dispersion of HMS $(75 \mu \mathrm{M})$ were mixed and homogenized in a glass vial and then irradiated with visible light from a $500-W$ mercury lamp. LHCs-b sample: $1 \mathrm{ml}$ of 4 -NP stock solution $(2 \mathrm{mM}), 100 \mu \mathrm{l}$ of sodium nitrite stock solution $(0.1 \mathrm{M})$ and $300 \mu \mathrm{l}$ of a dispersion of LHCs-b $(75 \mu \mathrm{M})$ were mixed and homogenized in a glass vial and then irradiated with visible light from a $500-\mathrm{W}$ mercury lamp.

Untreated control group. Blank sample: $1 \mathrm{ml}$ of 4-NP stock solution (2 mM), $100 \mu \mathrm{l}$ sodium nitrite stock solution $(0.1 \mathrm{M})$ and $300 \mu \mathrm{l}$ of distilled water were mixed and homogenized in a glass vial and then stored in the dark. $\beta$-CAR sample: $1 \mathrm{ml}$ of 4 -NP stock solution $(2 \mathrm{mM}), 100 \mu \mathrm{l}$ sodium nitrite stock solution $(0.1 \mathrm{M})$ and $300 \mu \mathrm{l}$ of a dispersion of $\beta$-CAR $(75 \mu \mathrm{M})$ were mixed and homogenized in a glass vial and then stored in the dark. Chl-b sample: $1 \mathrm{ml}$ of 4-NP stock solution $(2 \mathrm{mM}), 100 \mu \mathrm{l}$ sodium nitrite stock solution $(0.1 \mathrm{M})$ and $300 \mu \mathrm{l}$ of a dispersion of Chl-b $(75 \mu \mathrm{M})$ were mixed and homogenized in a glass vial and then stored in the dark. HMS sample: $1 \mathrm{ml}$ of 4 -NP stock solution $(2 \mathrm{mM}), 100 \mu \mathrm{l}$ sodium nitrite stock solution $(0.1 \mathrm{M})$ and $300 \mu \mathrm{l}$ of a dispersion of HMS $(75 \mu \mathrm{M})$ were mixed and homogenized in a glass vial and then stored in the dark. LHCs-b: $1 \mathrm{ml}$ of 4-NP stock solution $(2 \mathrm{mM}), 100 \mu \mathrm{l}$ sodium nitrite stock solution $(0.1 \mathrm{M})$ and $300 \mu \mathrm{l}$ of a dispersion of LHCs-b $(75 \mu \mathrm{M})$ were mixed and homogenized in a glass vial and then stored in the dark. Changes in the absorption peaks over time associated with the photocatalytic reduction of 4-NP to 4-AP (experimental group and untreated control group) were monitored by ultraviolet-visible spectroscopy (UV-2501)

For the photocatalytic activity investigation of the LHCs-b sample using a longer duration of irradiation, the procedure was as follows: A 4-NP stock solution $(2 \mathrm{mM})$, a sodium nitrite stock solution $(0.1 \mathrm{M})$ and a dispersion of LHCs-b were mixed and homogenized in a glass vial and then irradiated with visible light from a $300-\mathrm{W}$ Xe lamp. Changes in the absorption peaks over time associated with the photocatalytic reduction of 4-NP to 4-AP (0-120 min) were monitored by ultraviolet-visible spectroscopy (UV-2501).

Data availability. The authors declare that the data supporting the findings of this study are available within the article and its Supplementary Information files, and all relevant data are available from the authors.

\section{References}

1. Álvarez, R., Vaz, B., Gronemeyer, H. \& Lera, Á. R. Functions, therapeutic applications, and synthesis of retinoids and carotenoids. Chem. Rev. 114, 1-125 (2014).

2. Britton, G. Structure and properties of carotenoids in relation to function. FASEB J. 9, 1551-1558 (1995).

3. Chatgilialoglu, C., Ferreri, C., Melchiorre, M., Sansone, A. \& Torreggiani, A. Lipid geometrical isomerism: from chemistry to biology and diagnostics. Chem. Rev. 114, 255-284 (2014).

4. Vishnevetsky, M., Ovadis, M. \& Vainstein, A. Carotenoid sequestration in plants: the role of carotenoid associated proteins. Trends Plant Sci. 4, 232-235 (1999).

5. Edge, R., Land, E. J., McGarvey, D., Mulroy, L. \& Truscott, T. G. Relative one-electron reduction potentials of carotenoid radical cations and the interactions of carotenoids with the vitamin E radical cation. J. Am. Chem. Soc. 120, 4087-4090 (1998)

6. Blount, J. D., Metcalfe, N. B., Birkhead, T. R. \& Surai, P. F. Carotenoid modulation of immune function and sexual attractiveness in zebra finches. Science 300, 125-127 (2003).

7. EI-Agamey, A. \& McGarvey, D. Evidence for a lack of reactivity of carotenoid addition radicals towards oxygen: a laser flash photolysis study of the reactions of carotenoids with acylperoxyl radicals in polar and non-polar solvents. J. Am. Chem. Soc. 125, 3330-3340 (2003).

8. Havaux, M. Carotenoids as membrane stabilizers in chloroplasts. Trends Plant Sci. 3, 147-151 (1998).

9. Staleva, H. et al. Mechanism of photoprotection in the cyanobacterial ancestor of plant antenna proteins. Nat. Chem. Biol. 11, 287-291 (2015).

10. Leverenz, R. L. et al. A $12 \AA$ carotenoid translocation in a photoswitch associated with cyanobacterial photoprotection. Science 348, 1463-1466 (2015).

11. Pascal, A. A. et al. Molecular basis of photoprotection and control of photosynthetic light-harvesting. Nature 436, 134-137 (2005).

12. Lakshmi, K. V. et al. Pulsed high-frequency EPR study on the location of carotenoid and chlorophyll cation radicals in photosystem II. J. Am. Chem. Soc 125, 5005-5014 (2003).

13. Moise, A. R., Al-Babili, S. \& Wurtzel, E. T. Mechanistic aspects of carotenoid biosynthesis. Chem. Rev. 114, 164-193 (2014).

14. Foss, B. J., Noess, S. N., Sliwka, H.-R. \& Partali, V. Stable and highly waterdispersible, highly unsaturated carotenoid phospholipids-surface properties and aggregate size. Angew. Chem. Int. Ed. 42, 5237-5240 (2003).

15. Auweter, H. et al. Supramolecular structure of precipitated nanosize $\beta$-carotene particles. Angew. Chem. Int. Ed. 15, 2188-2191 (1999).

16. Anantachoke, N. et al. Fine tuning the production of nanosized $\beta$-carotene particles using spinning disk processing. J. Am. Chem. Soc. 128, 13847-13853 (2006).

17. Zouni, A. et al. Crystal structure of photosystem II from Synechococcus

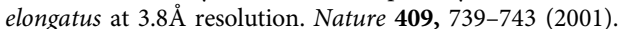

18. Qiu, Y., Chen, P. \& Liu, M. Evolution of various porphyrin nanostructures via an oil/ aqueous medium: controlled self-assembly, further organization, and supramolecular chirality. J. Am. Chem. Soc. 132, 9644-9652 (2010). 
19. Rufo, C. M. et al. Short peptides self-assemble to produce catalytic amyloids. Nat. Chem. 6, 303-309 (2014).

20. Tang, T-Y. D. et al. Fatty acid membrane assembly on coacervate microdroplets as a step towards a hybrid protocell model. Nat. Chem. 6, 527-533 (2014)

21. Calabrese, C. M. et al. Biocompatible infinite-coordination-polymer nanoparticle-nucleic-acid conjugates for antisense gene regulation. Angew. Chem. Int. Ed. 54, 476-480 (2015).

22. Liu, K. et al. Peptide-induced hierarchical long-range order and photocatalytic activity of porphyrin assemblies. Angew. Chem. Int. Ed. 54, 500-505 (2015)

23. Scholes, G., Fleming, G., Olaye-Castro, A. \& Grondelle, R. Lessons from nature about solar light harvesting. Nat. Chem. 3, 763-774 (2011).

24. Han, Y., Meng, Z., Ma, Y.-X. \& Chen, C.-F. Iptycene-derived crown ether hosts for molecular recognition and self-assembly. Acc. Chem. Res. 47, 2026-2040 (2014).

25. Wang, F. et al. Self-sorting organization of two heteroditopic monomers to supramolecular alternating copolymers. J. Am. Chem. Soc. 130, 11254-11255 (2008).

26. Yan, X. et al. A multiresponsive, shape-persistent, and elastic supramolecular polymer network gel constructed by orthogonal self-assembly. Adv. Mater. 24, 362-369 (2012).

27. Crini, G. Review: a history of cyclodextrins. Chem. Rev. 114, 10940-10975 (2014).

28. Zhang, H. \& Zhao, Y. Pillararene-based assemblies: design principle, preparation and applications. Chem. Eur. J 19, 16862-16879 (2013).

29. Shen, M., Sun, Y., Han, Y., Yao, R. \& Yan, C. Strong deaggregating effect of a novel polyamino resorcinarene surfactant on gold nanoaggregates under microwave irradiation. Langmuir 24, 13161-13167 (2008).

30. Xue, M., Yang, Y., Chi, X., Zhang, Z. \& Huang, F. Pillararenes, a new class of macrocycles for supramolecular chemistry. Acc. Chem. Res. 45, 1294-1308 (2012).

31. Yao, Y., Wang, Y. \& Huang, F. Synthesis of various supramolecular hybrid nanostructures based on pillar[6]arene modified gold nanoparticles/nanorods and their application in $\mathrm{pH}$ - and NIR-triggered controlled release. Chem. Sci. 5, 4312-4316 (2014).

32. Ogoshi, T. \& Yamagishi, T. Pillar [5]-and pillar[6]arene-based supramolecular assemblies built by using their cavity-size-dependent host-guest interactions. Chem. Commun. 50, 4776-4787 (2014).

33. Yu, G. et al. Pillar[6]arene/paraquat molecular recognition in water: high binding strength, ph-responsiveness, and application in controllable self-assembly, controlled release, and treatment of paraquat poisoning. J. Am. Chem. Soc. 134, 19489-19497 (2012).

34. Yu, G. et al. A water-soluble pillar[6]arene: synthesis, host-guest chemistry, and its application in dispersion of multiwalled carbon nanotubes in water. J. Am. Chem. Soc. 134, 13248-13251 (2012).

35. Yao, Y., Xue, M., Chen, J., Zhang, M. \& Huang, F. An amphiphilic pillar[5]arene: synthesis, controllable self-assembly in water, and application in calcein release and TNT adsorption. J. Am. Chem. Soc. 134, 15712-15715 (2012).

36. Chen, L. et al. Chiral selective transmembrane transport of amino acids through artificial channels. J. Am. Chem. Soc. 135, 2152-2155 (2013).

37. Duan, Q. et al. pH-responsive supramolecular vesicles based on water-soluble pillar[6]arene and ferrocene derivative for drug delivery. J. Am. Chem. Soc. 135, 10542-10549 (2013)

38. Ogoshi, T., Hashizume, M., Yamagishi, T. \& Nakamoto, Y. Synthesis, conformational and host-guest properties of water-soluble pillar[5]arene. Chem. Commun. 46, 3708-3710 (2010).

39. Cao, Y. et al. Supramolecular nanoparticles constructed by DOX-based prodrug with water-soluble Pillar[6]arene for self-catalyzed rapid drug release. Chem. Mater. 27, 1110-1119 (2015).

40. Wu, X., Li, Y., Lin, C., Hu, X. \& Wang, L. GSH- and pH-responsive drug delivery system constructed by water-soluble pillar[5]arene and lysine derivative for controllable drug release. Chem. Commun. 51, 6832-6835 (2015).

41. Takaya, T. \& Iwata, K. Relaxation mechanism of $\beta$-carotene from $\mathrm{S}_{2}\left(1 \mathrm{~B}_{\mathrm{u}}^{+}\right)$ State to $\mathrm{S}_{1}\left(2 \mathrm{Ag}^{-}\right)$state: femtosecond time-resolved near-ir absorption and stimulated resonance raman studies in 900-1550 nm region. J. Phys. Chem. A. 118, 4071-4078 (2014).

42. Oliveira, V. E. et al. Carotenoids and $\beta$-cyclodextrin inclusion complexes: Raman spectroscopy and theoretical investigation. J. Phys. Chem. A. 115, 8511-8519 (2011).

43. Schneider, H.-J. Dispersive interactions in solution complexes. Acc. Chem. Res. 46, 916-926 (2015).

44. Cao, Y. et al. Multistimuli-responsive supramolecular vesicles based on water-soluble pillar[6]arene and SAINT complexation for controllable drug release. J. Am. Chem. Soc. 136, 10762-10769 (2014).
45. Matsuzawa, Y. et al. Assembly and photoinduced organization of mono- and oligopeptide molecules containing an azobenzene moiety. Adv. Funct. Mater. 17, 1507-1514 (2007).

46. Spano, F. C. Analysis of the UV/Vis and CD spectral line shapes of carotenoid assemblies: spectral signatures of chiral $\mathrm{H}$-aggregates. J. Am. Chem. Soc. 131, 4267-4278 (2009).

47. Sun, Y., Yao, Y., Yan, C.-G., Han, Y. \& Shen, M. Selective decoration of metal nanoparticles inside or outside of organic microstructures via self-assembly of resorcinarene. ACS Nano 4, 2129-2141 (2010)

48. Sun, Y., Yan, C.-G., Yao, Y., Han, Y. \& Shen, M. Self-assembly and metallization of resorcinarene microtubes in water. Adv. Funct. Mater. 18, 3981-3990 (2008).

49. Soni, S. P., Ward, J. A., Sen, S. E., Feller, S. E. \& Wassall, S. R. Effect of trans unsaturation on molecular organization in a phospholipid membrane. Biochemistry 48, 11097-11107 (2009).

50. Li, C. et al. Self-assembly of [2] pseudorotaxanes based on pillar[5]arene and bis(imidazolium) cations. Chem. Commun. 46, 9016-9018 (2010).

51. Yamamoto, Y. et al. Photoconductive coaxial nanotubes of molecularly connected electron donor and acceptor layers. Science 314, 1761-1764 (2006)

52. Blau, W. J. \& Fleming, A. J. Designer nanotubes by molecular self-assembly. Science 304, 1457-1458 (2004).

53. Koehne, B., Elli, G., Jennings, R., Wilhelm, C. \& Trissl, H.-W. Spectroscopic and molecular characterization of a long wavelength absorbing antenna of Ostreobium sp. Biochim. Biophys. Acta. 1412, 94-107 (1999).

54. Hu, Z., Xu, Y., Gong, Y. \& Kuang, T. Effects of heat treatment on the protein secondary structure and pigment microenvironment in photosystem I complex. Photosynthetica 43, 529-534 (2005).

55. Yang, Z., Su, X., Wu, F., Gong, Y. \& Kuang, T. Effect of phosphatidylglycerol on molecular organization of photosystem I. Biophys. Chem. 115, 19-27 (2005).

56. Goss, R., Wilhelm, C. \& Garab, G. Organization of the pigment molecules in the chlorophyll a/b/c containing alga Mantoniella squamata (Prasinophyceae) studied by means of absorption, circular and linear dichroism spectroscopy. Biochim. Biophys. Acta. 1457, 190-199 (2000).

57. Dobrikova, A. et al. Structural rearrangements in chloroplast thylakoid membranes revealed by differential scanning calorimetry and circular dichroism spectroscopy. thermo-optic effect. Biochemistry. 42, 11272-11280 (2003).

58. Agostiano, A., Cosma, P., Trotta, M., Monsu-Scolaro, L. \& Micali, N Chlorophyll a behavior in aqueous solvents: formation of nanoscale self-asembled complexes. J. Phys. Chem. B 106, 12820-12829 (2002).

59. Kwa, S. et al. Steady-state and time-resolved polarized light spectroscopy of the green plant light-harvesting complex II. Biochim. Biophys. Acta. 1101, 143-146 (1992).

60. Dauthal, P. \& Mukhopadhyay, M. Prunus domestica fruit extract-mediated synthesis of gold nanoparticles and its catalytic activity for 4-nitrophenol reduction. Ind. Eng. Chem. Res. 51, 13014-13020 (2012).

61. Moran, N. \& Jarvik, T. Lateral transfer of genes from fungi underlies carotenoid production in aphids. Science 328, 624-628 (2010).

62. Valmalette, J. et al. Light-induced electron transfer and ATP synthesis in a carotene synthesizing insect. Sci. Rep. 2, 579 (2012).

63. Zou, Q. et al. Multifunctional porous microspheres based on peptideporphyrin hierarchical co-assembly. Angew. Chem. Int. Ed. 53, 2366-2370 (2014).

64. Mele, A., Mendichi, R., Selva, A., Molnar, P. \& Toth, G. Non-covalent associations of cyclomaltooligosaccharides(cyclodextrins) with carotenoids in water. A study on the $\alpha$-and $\beta$-cyclodextrin $/ \psi, \psi$-carotene (lycopene) systems by light scattering, ionspray ionization and tandem mass spectrometry. Carbohydr. Res. 337, 1129-1136 (2002).

65. Fernandez, J., Mellet, C. \& Defaye, J. Glyconanocavities: cyclodextrins and beyond. J. Inclusion Phenom. Macrocyclic Chem 56, 149-159 (2006).

66. Ashton, P. R. et al. Self-assembly, spectroscopic, and electrochemical properties of [n] rotaxanes1. J. Am. Chem. Soc. 118, 4931-4951 (1996).

\section{Acknowledgements}

This work was financially supported by the China Postdoctoral Science Foundation (137070124), the National Natural Science Foundation of China (21273195, 21503185), we thank J.G. Zheng of the University of California (Irvine) for help in TEM discussions, as well as F.M Zhang and Z.F. Wang for the NMR and SAXS experiments.

\section{Author contributions}

Y.S. and G.D. designed the experiments and analysed the data. ${ }^{1} \mathrm{H}-\mathrm{NMR}$, TEM, SEM, HRTEM, EDX mapping, DLS and CD investigations were performed by Y.S. F.G. and Y.S. designed and performed the fluorescence characterization and analysed the corresponding fluorescence data. Y.S. and T.Z. performed the optical microscopy and polarized optical microscopy experiments. Y.S. and J.H. performed the fluorescene 
optical microscopy experiments, FT-IR, and zeta-potential experiments. Y.S., T.Z., and J.H. performed the ultraviolet-visible and Raman spectra experiments. Y.S. and G.D. conceived the experiments and co-wrote the paper.

\section{Additional information}

Supplementary Information accompanies this paper at http://www.nature.com/ naturecommunications

Competing financial interests: The authors declare no competing financial interests.

Reprints and permission information is available online at http://npg.nature.com/ reprintsandpermissions/
How to cite this article: Sun, Y. et al. Stimulus-responsive light-harvesting complexes based on the pillararene-induced co-assembly of $\beta$-carotene and chlorophyll. Nat. Commun. 7:12042 doi: 10.1038/ncomms12042 (2016).

(c) (i) This work is licensed under a Creative Commons Attribution 4.0 International License. The images or other third party material in this article are included in the article's Creative Commons license, unless indicated otherwise in the credit line; if the material is not included under the Creative Commons license, users will need to obtain permission from the license holder to reproduce the material. To view a copy of this license, visit http://creativecommons.org/licenses/by/4.0/

(C) The Author(s) 2016 